\title{
Effect of Gaussian disorder on the voltage dependence of the current density in sandwich-type devices based on organic semiconductors
}

Citation for published version (APA):

Mensfoort, van, S. L. M., \& Coehoorn, R. (2008). Effect of Gaussian disorder on the voltage dependence of the current density in sandwich-type devices based on organic semiconductors. Physical Review B, 78(8), 0852071/16. [085207]. https://doi.org/10.1103/PhysRevB.78.085207

DOI:

10.1103/PhysRevB.78.085207

Document status and date:

Published: 01/01/2008

Document Version:

Publisher's PDF, also known as Version of Record (includes final page, issue and volume numbers)

Please check the document version of this publication:

- A submitted manuscript is the version of the article upon submission and before peer-review. There can be important differences between the submitted version and the official published version of record. People interested in the research are advised to contact the author for the final version of the publication, or visit the $\mathrm{DOI}$ to the publisher's website.

- The final author version and the galley proof are versions of the publication after peer review.

- The final published version features the final layout of the paper including the volume, issue and page numbers.

Link to publication

\footnotetext{
General rights

- You may freely distribute the URL identifying the publication in the public portal. follow below link for the End User Agreement:

www.tue.nl/taverne

Take down policy

If you believe that this document breaches copyright please contact us at:

openaccess@tue.nl

providing details and we will investigate your claim.
}

Copyright and moral rights for the publications made accessible in the public portal are retained by the authors and/or other copyright owners and it is a condition of accessing publications that users recognise and abide by the legal requirements associated with these rights.

- Users may download and print one copy of any publication from the public portal for the purpose of private study or research.

- You may not further distribute the material or use it for any profit-making activity or commercial gain

If the publication is distributed under the terms of Article $25 \mathrm{fa}$ of the Dutch Copyright Act, indicated by the "Taverne" license above, please 


\title{
Effect of Gaussian disorder on the voltage dependence of the current density in sandwich-type devices based on organic semiconductors
}

\author{
S. L. M. van Mensfoort and R. Coehoorn \\ Department of Applied Physics, Eindhoven University of Technology, P.O. Box 513, 5600 MB Eindhoven, The Netherlands \\ and Philips Research Laboratories, High Tech Campus 4, Box WAG-12, 5656 AE, Eindhoven, The Netherlands \\ (Received 23 August 2007; revised manuscript received 23 May 2008; published 13 August 2008)
}

\begin{abstract}
We investigate the effect of disorder on the voltage and layer thickness dependence of the current density in (metal/organic semiconductor/metal) devices containing organic semiconductors with a Gaussian shape of the density of states. The analysis is based on recently published numerically exact expressions for the dependence of the charge-carrier mobility on the carrier density and the electric field in such materials [W. F. Pasveer et al., Phys. Rev. Lett. 94, 206601 (2005)]. For the device simulations, a numerically efficient one-dimensional continuum drift-diffusion device model has been developed, which is also applicable to any other disorderinduced carrier density and field dependence of the mobility and diffusion coefficient. The device and material parameters chosen are relevant to organic light-emitting diode (OLED) applications. It is shown that a realistic degree of disorder can give rise to apparent mobilities that vary over more than 2 orders of magnitude with the layer thickness if the current-voltage curves are (incorrectly) analyzed in terms of the often-used drift-only Mott-Gurney formula. This implies that meaningful analyses of transport in OLEDs should be based on the full functional dependence of the mobility on the carrier density and field, induced by the disorder.
\end{abstract}

DOI: 10.1103/PhysRevB.78.085207

PACS number(s): 72.80.Le, 73.61.Ph, 85.30.De

\section{INTRODUCTION}

Devices that consist of an organic semiconductor which is sandwiched in between two metallic electrodes are intensively studied in view of applications in, for example, organic light-emitting diodes (OLEDs), ${ }^{1}$ organic photoconductors, ${ }^{2}$ and organic photovoltaic devices. ${ }^{3}$ The electron or hole mobility, $\mu$, of the organic semiconductors of which such devices are composed is frequently derived from the steady-state current density $(J)$ versus voltage $(V)$ characteristics of single-carrier devices, containing a single organic layer. ${ }^{4}$ The energy barriers at the interfaces for injection from the electrode into the organic semiconductor are then such that the charge carriers that are responsible for the current are either electrons or holes. In general, the injection barriers are not precisely equal at both interfaces, leading to a built-in voltage, $V_{\mathrm{bi}}$. In the absence of traps, and when (i) only the drift contribution to the current density is taken into account (neglecting the diffusion contribution), (ii) the barrier at the injecting electrode is insignificant, and (iii) the mobility may be assumed to be constant, the current density is for $V>V_{\text {bi }}$ given by the Mott-Gurney (MG) square law, ${ }^{5}$

$$
J_{\mathbf{M G}}=\frac{9}{8} \varepsilon \mu \frac{\left(V-V_{\mathrm{bi}}\right)^{2}}{L^{3}},
$$

with $\varepsilon$ and $L$ the permittivity and the thickness of the organic semiconductor, respectively.

In actual devices, deviations from Eq. (1) are found with a slope of $\log (J)$ versus $\log \left(V-V_{\mathrm{bi}}\right)$ curves smaller than 2 at relatively small voltages and higher than 2 at relatively large voltages. The former effect is a result of charge-carrier diffusion, which gives rise to the predominant contribution to the current density at small voltages and which even leads to a finite current density below $V_{\mathrm{bi}}$. At relatively large voltages, the drift contribution to the current density dominates. Recently, it has been demonstrated that energetic disorder in organic semiconductors used in OLEDs strongly affects the voltage dependence of the current density in both transport regimes. First, Tessler and co-workers ${ }^{6}$ showed that disorder can give rise to a diffusion coefficient enhancement above the value expected from the classical Einstein relation. For the specific case of Gaussian disorder, such an increase in the diffusion contribution to the current density occurs at high carrier densities. Second, Blom and co-workers ${ }^{7,8}$ demonstrated experimentally that the mobility in various materials that are frequently used in OLEDs, such as poly-( $p$-phenylene vinylene) (PPV), depends on the chargecarrier density. For organic field-effect transistors, a chargecarrier density dependence of the mobility had already been found earlier by Vissenberg and Matters, ${ }^{9}$ who explained the effect assuming an exponential density of states.

Roichman et al. ${ }^{10}$ were the first to include both effects of disorder on the mobility and the diffusion coefficient in a transport model for single-carrier sandwich-type devices. However, the model used in Ref. 10 for quantifying the carrier density dependence of the mobility was shown to neglect the percolative nature of the hopping transport, ${ }^{11}$ which strongly affects the temperature dependence of the mobility. Pasveer et al. ${ }^{12}$ developed a model that correctly includes the effects of percolation on the mobility and demonstrated that in the drift-dominated high-voltage regime the temperature dependence of the current density in PPV-based hole-only devices can be described well assuming Gaussian disorder. In their device model, the diffusion contribution to the current density was neglected. Although the effects of diffusion have been included in inorganic semiconductor device models, ${ }^{13}$ in models for OLEDs ${ }^{10,14}$ and in models for other organic electronic devices, ${ }^{15}$ so far in none of these studies the recent insights on the mobility in an organic semiconductor with Gaussian disorder, mentioned above, have been taken into account.

In this paper, we analyze the electrical transport through single-carrier (metal/organic semiconductor/metal) devices 
based on organic semiconductors with Gaussian disorder using a fully general model which includes the two effects of disorder mentioned above: the enhancement of the diffusion coefficient and the carrier density and field dependence of the mobility. This allows us to provide a complete description of the current-voltage curves. We have developed a practical method for calculating $J(V)$ curves based on a numerically fast one-dimensional continuum approach introduced by Bonham and Jarvis (BJ). ${ }^{16,17}$ The discussion of our method for extending the BJ approach is focused on applications to devices based on materials with a Gaussian density of states (DOS). However, the methodology is generic and is applicable to any type of disorder, provided that the carrier density and field dependences of the mobility and diffusion coefficient are known.

Within our model, applied to hole transport, we assume that at both electrodes thermal equilibrium is established between the electrode and the highest occupied molecular orbital (HOMO) of the organic semiconductor. This may be expected to yield a fair description of the transport physics when the injection barrier at the injecting contact is sufficiently small. Under these conditions, an appreciable space charge builds up at the injecting electrode, and the net current density at that electrode is the result of a diffusive contribution away from that electrode and a drift contribution toward that electrode. The underlying assumption is that the current density is not limited by the hopping rate of holes from states near the Fermi level in the metal to HOMO states on organic molecules close to the metal but by the finite bulk mobility. Our model thus does not address cases with a very high barrier at the injecting electrode, such as discussed by Campbell Scott and Malliaras ${ }^{18}$ for devices with a manifold of HOMO states with negligible width and by Arkhipov et $a l .{ }^{19}$ for the case of devices with a Gaussian DOS with a width larger than the thermal energy $k_{B} T$.

In its generic form, our model is also applicable to cases in which polaron formation has a more important effect on the mobility than energetic disorder. This will be the case when the polaron binding energy, $E_{\mathrm{pol}}$, is large as compared to the width, $\sigma$, of the Gaussian DOS. For that situation, Fishchuk et $a l .{ }^{20}$ have recently given an expression for the mobility. Polaron formation has previously been argued to determine the mobility in certain organic materials. ${ }^{21}$ In such a case, the mobility is essentially independent of the carrier concentration for concentrations below 0.1. ${ }^{20}$ However, as discussed above, there is strong experimental evidence that for the prototype material PPV, a polaron model does not provide a good description. This is consistent with results from a theoretical study by Meisel et al. ${ }^{22}$ who showed that for PPV the polaron binding energy, $E_{\mathrm{pol}}$, is very small $(<0.05 \mathrm{eV})$ as compared to the width, $\sigma$, of the Gaussian DOS $(\sim 0.14 \mathrm{eV})$, as deduced from an analysis of temperature dependent current-voltage curves. ${ }^{12}$

We analyze in detail how Gaussian disorder affects the full $J(V)$ curves of symmetric devices $\left(V_{\mathrm{bi}}=0 \mathrm{~V}\right)$ with excellently injecting contacts, and the $J(V)$ curves of devices with one excellently injecting electrode and one electrode which gives rise to a high injection barrier (resulting in a large value of $V_{\mathrm{bi}}$ ). A key result of our study is that (conventional) analyses of $J(V)$ curves using the Mott-Gurney expression given by Eq. (1) can, incorrectly, lead to a very strong layer thickness dependence of the apparent mobility. The effect increases with increasing disorder.

Section II contains the description of the carrier density and field-dependent mobility in a semiconductor with Gaussian disorder, used in this paper, and a brief description of the extended Bonham-Jarvis method, applied to devices based on such semiconductors. More technical discussions, including derivations of the expressions for the drift-diffusion equation and the current density in terms of dimensionless quantities, the iterative methods, used and the parametrization of the mobility and diffusion coefficient used, are given in four Appendixes A-D. In Sec. III, the effects of Gaussian disorder on the $J(V)$ curves are discussed for symmetric devices and for devices with a large built-in voltage. Section IV contains a summary, conclusions, and outlook.

\section{CAlCUlational METHOD}

\section{A. Mobility and diffusion coefficient in a material with a Gaussian DOS}

We focus in this paper on transport in devices containing an organic semiconductor with Gaussian disorder. The semiconductor is characterized by three parameters: the site density, $N_{t}$, the width of the DOS, $\sigma$, and the inverse wavefunction localization length, $\alpha$. In small-molecule organic semiconductors and in polymers, $N_{t}$ may be associated with the number of molecules and with the number of conjugated segments per volume unit, respectively. Also the width, $\sigma$, is a physically well-defined parameter, at least for smallmolecule materials, which can be deduced from the modeling of a sufficiently extended set of temperature and layer thickness dependent $J(V)$ curves. $^{23}$ Typical values of $\sigma$ in disordered organic materials are $0.08-0.15 \mathrm{eV}$. The inverse localization length, $\alpha$, describes in an effective way how the hopping rate between two sites decreases with increasing distance $R$, viz., as $\exp (-2 \alpha R)$ (see Ref. 12). For organic semiconducting materials, the average intersite distance $a=N_{t}^{-1 / 3}$ and the wave-function localization length are typically of the order of 1 and $0.1 \mathrm{~nm}$, respectively, ${ }^{24-26}$ so that $\alpha^{-1} \approx 0.1$ $\times a$. The energy levels at neighboring sites are assumed to be uncorrelated. The Gaussian DOS is given by

$$
N(E)=\frac{N_{t}}{\sqrt{2 \pi \sigma^{2}}} \exp \left(-\frac{E^{2}}{2 \sigma^{2}}\right) .
$$

As shown by Pasveer et al., ${ }^{12}$ the dependence of the mobility on the carrier density, $n$, and the field, $F$, can then be factorized so that

$$
\mu(T, n, F)=\mu_{0}(T) \times g_{1}(n, T) \times g_{2}(F, T) .
$$

Here $\mu_{0}(T)$ is the temperature $(T)$-dependent mobility in the limit of a zero carrier density and zero electric field, and $g_{1}$ and $g_{2}$ are dimensionless carrier density and field-dependent mobility enhancement factors, respectively. The diffusion coefficient, $D$, is given by the generalized Einstein equation, ${ }^{6}$ 

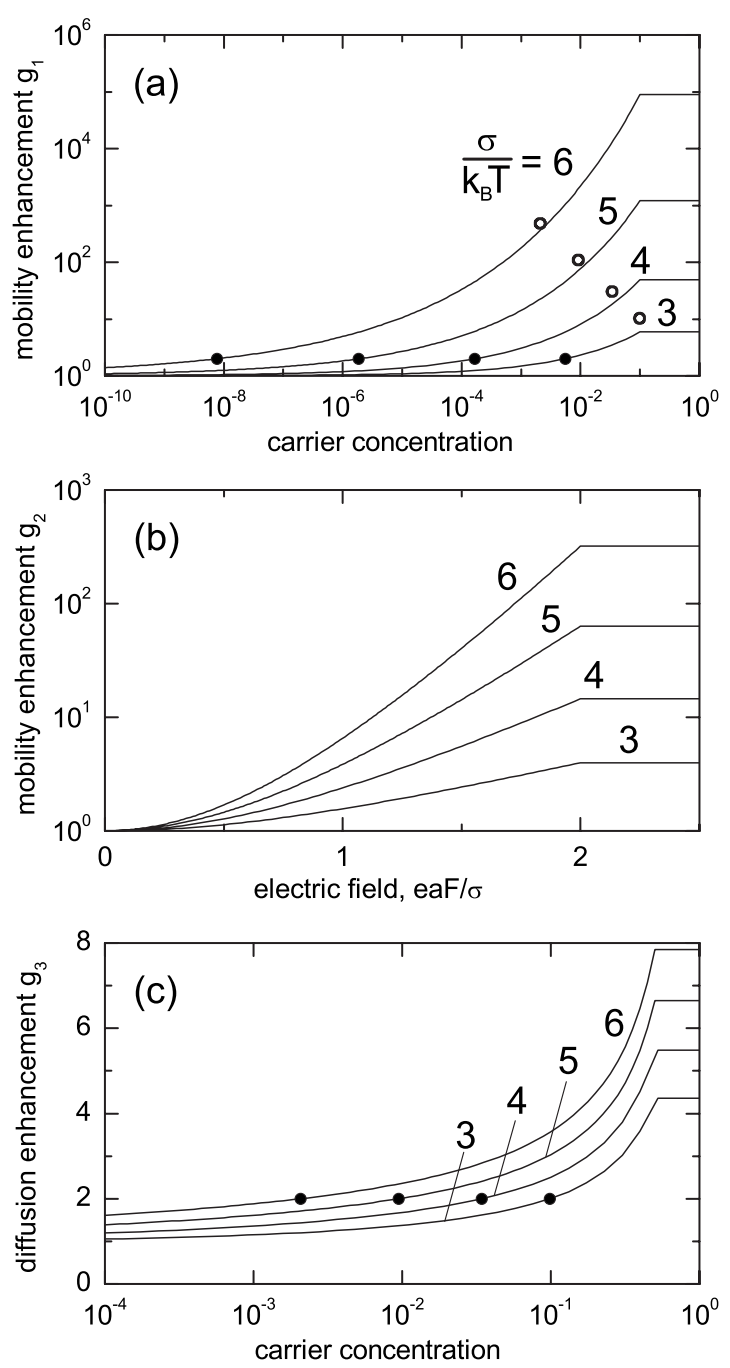

FIG. 1. Enhancement functions for a Gaussian DOS with dimensionless widths $\sigma /\left(k_{B} T\right)=3,4,5$, and 6, as defined in Appendix A: (a) $g_{1}$ as a function of the carrier concentration, $n / N_{t}$, (b) $g_{2}$ as a function of the reduced field $e a F / \sigma$, and (c) $g_{3}$ as a function of the carrier concentration. The filled circles in (a) and (c) indicate the concentrations $c^{*}$ and $c^{* *}$, respectively, where the mobility and diffusion coefficient, respectively, are enhanced by a factor 2 (see also Appendix A). The open circles in (a) indicate the approximate values $g_{1} \approx 1 / c^{* *}$ at the concentrations $c^{* *}$.

$$
D(T, n, F)=\frac{k_{B} T}{e} \mu(T, n, F) \times g_{3}(T, n),
$$

where $k_{B}$ is the Boltzmann constant and $g_{3}$ is a dimensionless diffusion coefficient enhancement function that follows from the shape of the density of states. We make use of the compact expressions for the functions $g_{1}$ and $g_{2}$ that have been given for the value of $\alpha^{-1}=0.1 \times a$ used in Ref. 12 with a cutoff at high carrier densities in order to obtain a better agreement with the numerically exact results given in Ref. 12. The expressions used for $g_{1}, g_{2}$, and $g_{3}$ are given in Appendix A. Figures 1(a)-1(c) show the dependence of $g_{1}$ and $g_{3}$ on the carrier concentration and of $g_{2}$ on the field for various values of $\sigma /\left(k_{B} T\right)$.

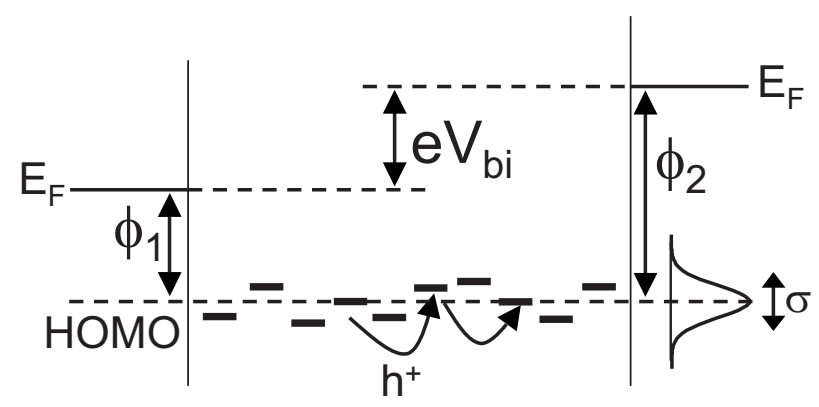

FIG. 2. Schematic energy diagram of a hole-only (metal/organic semiconductor/metal) device with a Gaussian distribution with width $\sigma$ of HOMO states and with $\varphi_{1}$ and $\varphi_{2}$ the hole injection barriers at the left and right interfaces. $E_{F}$ is the Fermi level in the metallic electrodes and $V_{\mathrm{bi}}$ is the built-in voltage. The arrows indicate schematically the hole $\left(h^{+}\right)$hopping.

\section{B. Method for solving the drift-diffusion equation}

We consider hole transport in single-layer devices with electrode interfaces at $x=0$ and $x=L$, where the carrier densities are $n_{1} \equiv n(0)$ and $n_{2} \equiv n(L)$, respectively. A schematic description of the energy-level structure assumed is shown in Fig. 2. The injection barriers at the left and right electrodes, $\varphi_{1}$ and $\varphi_{2}$, respectively, are defined as the difference between the Fermi energy in the metallic electrode and the top of the Gaussian DOS of the HOMO states. The built-in voltage is thus equal to $V_{\mathrm{bi}}=\left(\varphi_{2}-\varphi_{1}\right) / e$, with $e$ the elementary charge. By definition, for positive voltages, holes move from electrode 1 to electrode 2 . In the bulk of the device, transport takes predominantly place via hops in the tail of the Gaussian DOS. At the electrode interfaces we assume thermal equilibrium so that the carrier density at interface $i$ is given by

$$
n_{i}=\int_{-\infty}^{\infty} N(E) \frac{1}{1+\exp \left(\frac{E+\phi_{i}}{k_{B} T}\right)} d E .
$$

The current density is a sum of drift and diffusion contributions,

$$
J=e \mu(x) n(x) F(x)-e D(x) \frac{d n(x)}{d x} .
$$

The field and carrier densities are related via the Poisson equation,

$$
\varepsilon \frac{d F(x)}{d x}=e n(x),
$$

with $\varepsilon$ the permittivity.

Bonham and Jarvis ${ }^{16,17}$ developed an efficient method for solving the drift-diffusion problem for the case of a constant (position independent) mobility and diffusion coefficient. The basic merit of their method is that the two-point boundary-value problem is reformulated as an initial value problem: the solution of the drift-diffusion equation is developed from a single known starting point. Use is made of the fact that the Poisson equation implies that the field increases monotonically with position. It is therefore possible to trans- 


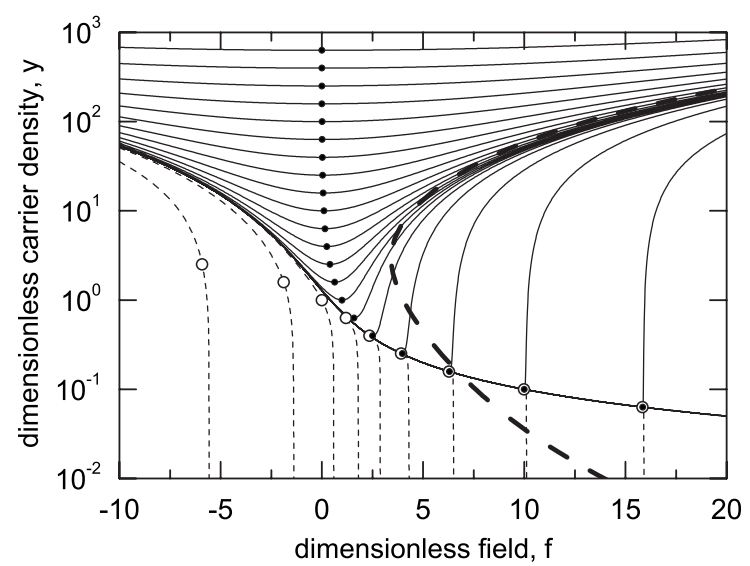

FIG. 3. Solutions $y(f)$ of the dimensionless transport equation [Eq. (8)] for the case of a constant mobility for a range of equidistant values of the minimum dimensionless carrier density $y_{\min }$ (full curves, minimum positions indicated by filled circles, type-I solutions) and for a range of equidistant values of carrier densities at which inflection points $y_{i}$ are obtained (dashed curves, inflection points indicated by open circles, type-II solutions). The thick dashed line connects the second electrode end points, $y_{2}\left(f_{2}\right)$, of segments of the $y(f)$ curves which are a solution of Eq. (8) for the case $y_{1}=10^{4} \times y_{2}$, studied in Fig. 4(b), for a range of values of the current density (see also Appendix D).

form Eq. (6) into an expression within which the $x$ dependence of $n$ is replaced by an $F$ dependence. For convenience, Bonham and Jarvis ${ }^{16,17}$ furthermore proposed a transformation in order to make all quantities dimensionless. We have extended the BJ method to include disordered systems with a carrier density and field-dependent mobility. As shown in Appendix B, Eq. (6) can then be transformed to the following dimensionless transport equation,

$$
\frac{d y}{d f}=\frac{f}{g_{3}[n(y)]}-\frac{1}{g_{1}[n(y)] \times g_{2}[F(f)] \times g_{3}[n(y)] \times y},
$$

with $y$ the dimensionless carrier density and $f$ the dimensionless field. The transformations from $n$ to $y$ and from $F$ to $f$ are included in Appendix B.

For the case of a constant mobility and diffusion coefficient, Eq. (8) simplifies to $d y / d f=f-1 / y$ as $g_{1}=g_{2}=g_{3}=1$. The solutions are shown in Fig. 3. The curves show, strictly speaking, how the carrier density can vary as a function of the field in the device. However, the curves also tell how, more qualitatively, the carrier density can vary with the position across the device, in view of the monotonous increase of the field with position, explained above. The field-position relationship is given in Appendix B [Eq. (B13)]. It is apparent that there are two types of curves, as noticed first by Bonham and Jarvis. ${ }^{16,17}$ The full curves in the upper part of the figure ("type-I solutions"), all show a (positive) minimum value at a certain value of $f$. The dashed curves in the lower part of the figure ("type-II solutions") show no minimum, and at a certain value of $f, y(f)$ equals zero. All solutions of the dimensionless transport equation [Eq. (8)] fall under these two categories. The solution is always of type I if the injection barriers at both interfaces are equal so that $n_{1}$ $=n_{2}$ and $V_{\mathrm{bi}}=0$, as there is then a minimum at any voltage. Otherwise, the solution is only of type $\mathrm{I}$ if $n_{1}$ and $n_{2}$ are sufficiently large and the voltage is sufficiently small. The distinction between these two solution types remains valid in the case of a Gaussian DOS.

Following the method introduced by Bonham and Jarvis, ${ }^{16,17}$ the type-I solutions are obtained as follows. For a selected value of the dimensionless carrier density at the minimum, $y_{\min }$, the dimensionless field at the minimum, $f_{\min }$, follows by setting the right-hand part of Eq. (8) to zero. For the case of a constant mobility and diffusion coefficient, $f_{\min }=1 / y_{\min }$. Subsequently, the function $y(f)$ can be obtained using Eq. (8) by numerical integration toward smaller and larger $f$ using, e.g., a Runge-Kutta method. The integration can then be stopped when the dimensionless boundary carrier densities $y_{1}$ and $y_{2}$ have been reached at fields $f_{1}$ and $f_{2}$, respectively. The backtransformation of the resulting function $y(f)$ in the interval $\left[f_{1} ; f_{2}\right]$ to the carrier density across the device, and expressions for the current density and voltage in terms of $y(f)$ are given in Appendix B. A complication is that the relationship between the dimensionless boundary carrier densities $y_{1}$ and $y_{2}$ and the actual values $n_{1}$ and $n_{2}$ contains the-yet unknown-value of the current density [see Eqs. (B1) and (B9)]. Therefore, the solution has to be determined by an iterative method, as explained in detail in Appendix C. $J(V)$ curves can be obtained by repeating these calculations for a series of $f_{\min }$ values.

For obtaining the type-II solutions, points $\left(f=f_{0}, y=0\right)$ may be used as starting points for the integration, as explained by Bonham and Jarvis. ${ }^{17}$ In this case, only a single integration, toward the value $f=f_{1}\left(<f_{0}\right)$ at which $y=y_{1}$, is needed. In the same way as described for type-I solutions, the carrier density and current density can then be obtained from $y(f)$, calculated in the interval $\left[f_{1}\left(y_{1}\right), f_{2}\left(y_{2}\right)\right]$.

In practice, this approach may lead to numerical difficulties, as at the starting point of the integration the slope of $y(f)$ diverges [see Eq. (8) and Fig. 3]. Therefore, we propose the use of an alternative starting point for the integration procedure. We have found that all type-II curves have an inflection point, $\left(f_{i}, y_{i}\right)$, at which $d^{2} y / d f^{2}=0$. In Appendix B, an implicit relationship between $f_{i}$ and $y_{i}$ is given [Eq. (B14)], from which $y_{i}$ can be obtained from a root-search procedure for a given value of $f_{i}$. For the case of a constant mobility and diffusion coefficient, $f_{i}=1 / y_{i}-y_{i}^{2}$. The function $y(f)$ can then be obtained by using the inflection point as the starting point of numerical integration toward smaller and larger $f$ until the points at which $y(f)=f_{1}$ and $f_{2}$, respectively, are reached. We find that a further improvement of the efficiency of the method is obtained by calculating $f(y)$ functions, instead of $y(f)$ functions. Technical difficulties related to the divergence of the slope of the $y(f)$ curves when $y$ approaches zero are then avoided. The expressions that are used for obtaining the current density and voltage from the calculated $f(y)$ function are included in Appendix B. In Appendix D some remarks are given concerning the voltage at which the transition between type-I and type-II solutions takes place. 


\section{APPLICATION TO DEVICES WITH A GAUSSIAN DOS}

In this section we present and analyze the dependence of the $J(V)$ curves on the width of the Gaussian DOS. In Sec. III A, the effect of disorder on the carrier density across the device is shown, and the transition from type-I to type-II solutions is illustrated. In Secs. III B and III C, on symmetric and asymmetric devices, with $V_{\mathrm{bi}}=0$ and $V_{\mathrm{bi}}>0$, respectively, it is shown how the introduction of disorder affects the voltage dependence of the current density in the diffusion dominated and drift dominated transport regimes and at the crossover between the two regimes. Furthermore, it is demonstrated that, as a result of disorder, the apparent mobility that would follow from a conventional analysis of the $J(V)$ curves using the drift-only Mott-Gurney relation [Eq. (1)] can vary over many orders of magnitude if the device thickness is varied.

\section{A. Carrier density}

In Fig. 4, the effect of disorder on the voltage dependent carrier density at $T=298$ across (a) one symmetric and [(b) and (c)] two asymmetric devices is shown. The full and dashed curves show the carrier density for the case of a disordered material, with $\sigma /\left(k_{B} T\right)=6$, and for the case of a material with a constant mobility and diffusion coefficient, respectively. The latter situation would occur if at all points across the device the transport would be in the Boltzmann transport regime, which is the case, e.g., when the DOS is a $\delta$ function at an energy more than a few times $k_{B} T$ above the Fermi level of the two electrodes. The mobility and the diffusion coefficient are then related by the standard Einstein equation [i.e., the factor $g_{3}$, defined by Eq. (A5), is equal to $1]$.

In the limiting case of a symmetric device with ideal contacts $\left(n_{1}=n_{2}=\infty\right)$ and with a constant mobility and diffusion coefficient, the carrier density at $V=0$ is given by ${ }^{27}$

$$
n(x)=\frac{2 \pi^{2}}{\cos ^{2}\left[\pi\left(\frac{x}{L}-\frac{1}{2}\right)\right]} \times n_{0},
$$

with

$$
n_{0}=\frac{\varepsilon k_{B} T}{e^{2} L^{2}} .
$$

It follows that the carrier density in the device center is then equal to $n_{\text {center }}=2 \pi^{2} \times n_{0}$. For all devices considered in Figs. 4(a)-4(c), the carrier density at the left electrode is taken to be equal to $n_{1}=10^{6} \times n_{0}$. This ensures that injection at that electrode can be characterized as "excellent" as at the left contact the carrier density is then many orders of magnitude larger than $n_{\text {center }}$. We take $L=100 \mathrm{~nm}$ and $\varepsilon_{r}=3$ (relative permittivity) so that $n_{1}=4.25 \times 10^{26} \mathrm{~m}^{-3}$. This is of the order of the site density in a typical organic semiconductor $\left(\sim 10^{27} \mathrm{~m}^{-3}\right)$. For the systems with Gaussian disorder, we assume that at the left interface the Fermi level coincides with the top of the Gaussian DOS. This implies that the site density is given by $N_{t}=2 n_{1}=8.51 \times 10^{26} \mathrm{~m}^{-3}$.

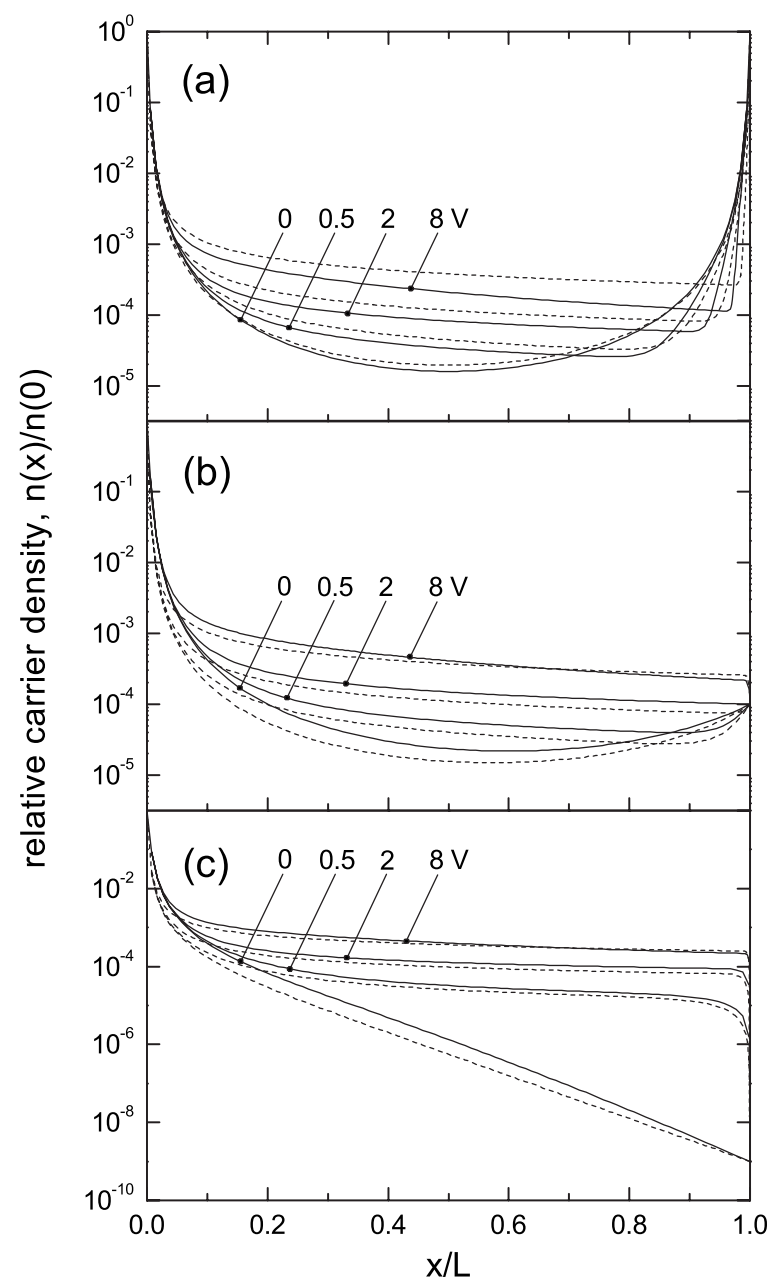

FIG. 4. Calculated carrier density, with respect to the carrier density at $x=0$, at $T=298 \mathrm{~K}$, and at various voltages for devices with a Gaussian DOS with width $\sigma$ such that $\sigma /\left(k_{B} T\right)=6$ (full curves) and for the case of a constant mobility (dashed curves). In all cases, $L=100 \mathrm{~nm}, n_{1}=4.25 \times 10^{26} \mathrm{~m}^{-3}, N_{t}=8.51 \times 10^{26} \mathrm{~m}^{-3}$ (for systems with a Gaussian DOS), $\mu_{0}=1.0 \times 10^{-10} \mathrm{~m}^{2} /(\mathrm{Vs})$, $\varepsilon_{r}=3$, and (a) $n_{2}=n_{1}$, (b) $n_{2}=10^{-4} \times n_{1}$, and (c) $n_{2}=10^{-9} \times n_{1}$.

Figure 4(a) shows that for symmetric devices, with $n_{1}$ $=n_{2}$ so that $V_{\mathrm{bi}}=0 \mathrm{~V}$, the carrier density shows a minimum at all voltages (type-I solutions). For the case of a constant mobility and diffusion coefficient, the relative carrier density at $V=0$ in the device center, $n_{\text {center }} / n_{1}$ is not significantly different from the value $2 \pi^{2} \times 10^{-6}$ expected for the case of ideal contacts using Eq. (9). For the case of a disordered material, we do not have an analytical expression for $n(x)$. The numerical results shown by the figure reveal that the effect of disorder on the carrier density across the devices is surprisingly small. For $V=0$, the system is in thermal equilibrium so that the small difference is not related to the detailed form of the density and field dependence of the mobility but only to the different carrier density dependences of the $g_{3}$ functions (with the factor $g_{3}$ as given by the generalized Einstein equation [Eq. (A5)] for the case of a Gaussian DOS and with $g_{3}=1$ assumed here for the case of a constant mobility). Comparison of the full and dashed curves in Fig. 4(a) reveals that for all voltages the introduction of disorder 
leads to a larger carrier density near the electrodes and a smaller carrier density in the device center. We view the former effect as the result of the enhanced diffusion coefficient, and the latter effect as the self-consistent result of the enhanced space charge near the electrodes, which counteracts diffusion to points deep inside the device.

Figure 4(b) shows the carrier density in devices with a carrier density at the right contact equal to $10^{-4} n_{1}$. For the case of a constant mobility and diffusion coefficient, $V_{\mathrm{bi}}$ $=\left(k_{B} T / e\right) \times \ln \left(n_{1} / n_{2}\right)$, so that $V_{\mathrm{bi}}=0.24 \mathrm{~V}$. For devices with disorder, $V_{\mathrm{bi}}$ is equal to the difference of the Fermi-level energies in a Gaussian DOS with $\sigma /\left(k_{B} T\right)=6$ that correspond to the carrier densities $n_{1}$ and $n_{2}$, leading to $V_{\mathrm{bi}}=0.60 \mathrm{~V}$. The value of $n_{2}$ used in this figure is still much larger than $2 \pi^{2}$ $\times n_{0}$. At small voltages, the carrier density shows therefore still a minimum. However, for sufficiently large voltages, above a transition voltage $V^{*}, n(x)$ decreases monotonically with increasing $x$. At small voltages, the introduction of disorder leads to an increase in the carrier density at all positions across the device. The effect of disorder is opposite to that for symmetric devices, shown in Fig. 4(a), because there is no large space charge near the second contact which can strongly hinder the increase in $n(x)$ due to diffusion from the first contact. As a result, the transition voltage revealed by Fig. 4(b) between the type-I and type-II solutions is smaller for the case of disorder with $\sigma /\left(k_{B} T\right)=6\left(V^{*}=2.00 \mathrm{~V}\right)$ than for the case of a constant mobility and diffusion coefficient $\left(V^{*}=2.86 \mathrm{~V}\right)$.

When the carrier density at the second electrode decreases, $V^{*}$ decreases until it becomes zero when $n_{2}$ is sufficiently small. Such a situation is shown in Fig. 4(c), which gives the carrier density for the case $n_{2}=10^{-9} n_{1}$, so that $V_{\mathrm{bi}}$ $=0.54$ and $0.98 \mathrm{~V}$ for devices without and with disorder, respectively. At all voltages, the carrier density decreases then monotonically with increasing $x$ (type-II solutions). For devices with one ideal contact $\left(n_{1}=\infty\right)$, and a second contact with a high injection barrier $\left(n_{2} / n_{0} \ll 1\right)$, and in the case of a constant mobility and diffusion coefficient, the carrier density is given by ${ }^{27}$

$$
n(x)=\frac{2 A^{2}}{\sinh ^{2}\left(A \frac{x}{L}\right)} \times n_{0},
$$

where the parameter $A$ (which is much larger than 1) follows from the application of the boundary condition at the second contact. At positions not too close to the first electrode, the carrier density is thus to an excellent approximation given by $n(x)=n_{0} \times 8 A^{2} / \exp (2 A x / L)$. This explains the linear $x$ dependence of $\log _{10}[n(x)]$ for $x / L>0.2$ shown in Fig. 4(c) for the case $V=0$. As in Fig. 4(b), the effect of disorder on $n(x)$ is opposite to that for symmetric devices, shown in Fig. 4(a). This may be explained in the same manner as discussed above for Fig. 4(b).

Below $8 \mathrm{~V}$, the carrier density shows a steady increase with voltage throughout almost the entire device. However, this trend is disrupted above $8 \mathrm{~V}$. As an example, the thin and thick full lines in Fig. 5(a) show the carrier densities at 8 and $16 \mathrm{~V}$, respectively, for the symmetric devices discussed

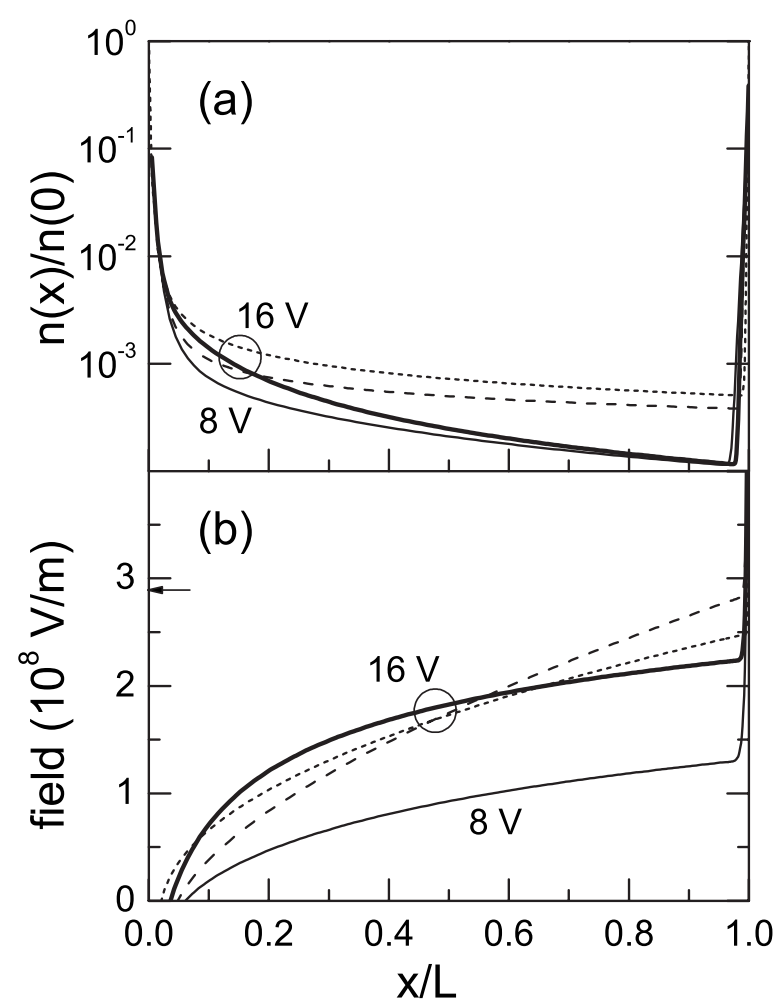

FIG. 5. (a) Reduced carrier density and (b) field across a symmetric device with $\sigma /\left(k_{B} T\right)=6$ and with the other parameters as in Fig. 4(a), at 8 and $16 \mathrm{~V}$ (thin and thick full curves, respectively). For $V=16 \mathrm{~V}$, the long-dashed and short-dashed curves give results obtained by switching off the field dependence of the mobility $\left(g_{2}\right.$ $=0$ ) and obtained for a constant mobility, respectively. The arrow indicates the cutoff field (see Appendix A).

above [Fig. 4(a)]. The carrier density shows for $x>L / 2$ only a very small increase, much less then shown in Fig. 4(a) when varying $V$ from 2 to $8 \mathrm{~V}$ and close to the second electrode even a decrease. This effect can be attributed to a strong field enhancement of the mobility in this part of the device so that a given local current density can already be obtained for a smaller local carrier density. Figure 5(b) shows that for $V=16 \mathrm{~V}$, the electric field is very high in the region $x>L / 2$ and even approaching $F_{\text {cutoff }}(2.8$ $\left.\times 10^{8} \mathrm{~V} / \mathrm{m}\right)($ see Appendix A). An explicit proof of the strong effect of the field enhancement of the mobility has been obtained by carrying out a calculation for $V=16 \mathrm{~V}$ with $g_{2}=0$. The long-dashed curve in Fig. 5(a) shows that switching off the field enhancement of the mobility results in a much larger carrier density for $x>L / 2$, approaching the values for devices without disorder (short-dashed curve). Furthermore, Fig. 5(b) shows that at $16 \mathrm{~V}$ the full effect of disorder (including the field dependence of the mobility) is a decrease in the field near the second electrode (thick full curve) with respect to the case without disorder (shortdashed curve), whereas the bare effect of the carrier density dependence of the mobility due to disorder is an increase in the field near the second electrode (long-dashed curve). This increase occurs because the enhanced mobility in the highdensity region near the first electrode is self-consistently matched by an increase in the field in the lower-density re- 


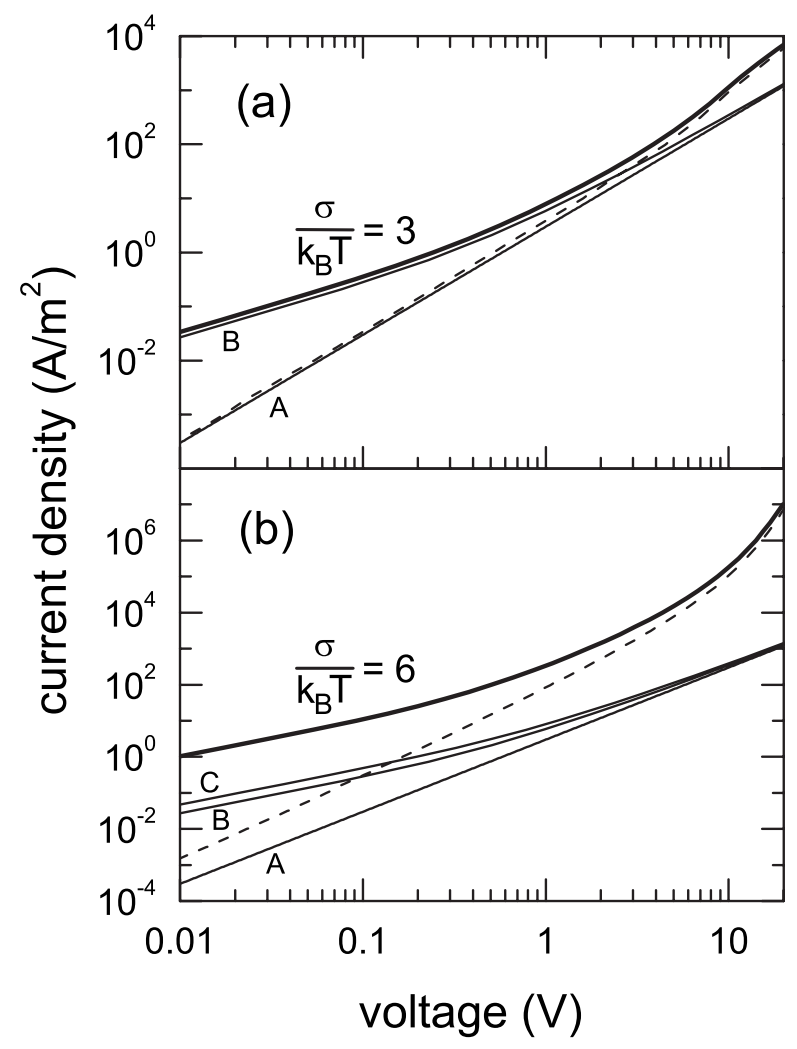

FIG. 6. $J(V)$ curves for transport in a Gaussian DOS in symmetric devices at $T=298 \mathrm{~K}$ with $L=100 \mathrm{~nm}, \quad n_{1}=n_{2}=2.13$ $\times 10^{26} \mathrm{~m}^{-3}, N_{t}=4.25 \times 10^{26} \mathrm{~m}^{-3}, \mu_{0}=1.0 \times 10^{-10} \mathrm{~m}^{2} /(\mathrm{Vs})$, and $\varepsilon_{r}=3$ and with (a) $\sigma /\left(k_{B} T\right)=3$ and (b) $\sigma /\left(k_{B} T\right)=6$. The thick curves give the result including disorder and diffusion. Curves $\mathrm{A}$ and $\mathrm{B}$ give the results for a constant mobility and without [Eq. (1)] and with diffusion, respectively. Curve $\mathrm{C}$ in (b) gives the drift-diffusion result for a system with $\sigma /\left(k_{B} T\right)=6$, but only taking the diffusion coefficient enhancement into account. The dashed curves give the results including disorder but without diffusion.

gion near the second electrode. A similar difference between the fields across the device was found by Tanase et al. ${ }^{8}$ when making for drift-only systems a comparison for the cases of a carrier density and a field-dependent mobility (Fig. 3 in Ref. 8).

\section{B. Current density in symmetric devices $\left(V_{\mathrm{bi}}=0\right)$}

In Fig. 6, the effect of disorder on the voltage dependence of the current density in symmetric devices at $298 \mathrm{~K}$ is shown. We take $L=100 \mathrm{~nm}, \varepsilon_{r}=3$, and $n_{1}=n_{2}=2.13$ $\times 10^{26} \mathrm{~m}^{-3}\left(n_{1} / n_{0}=0.5 \times 10^{6}\right)$. The thick full lines show the results for devices with Gaussian disorder with $\sigma /\left(k_{B} T\right)=3$ and 6 in Figs. 6(a) and 6(b), respectively. The calculations were performed assuming densities $N_{t}=2 n_{1}$ so that at the interfaces the Fermi energy coincides with the top of the Gaussian DOS. A first thin full curve in the figures shows the current density for a constant mobility and neglecting diffusion [Mott-Gurney formula, Eq. (1), curves A]. A second thin full curve shows how, for the case of a constant mobility and diffusion coefficient, the current density is enhanced above the Mott-Gurney result if diffusion is included (curves B). In
Fig. 6(b), a third thin full curve gives the current density including the enhancement of the diffusion coefficient, as given by Eq. (4), for the case of a Gaussian DOS with $\sigma /\left(k_{B} T\right)=6$ (curve C). The dashed curves give the current density in a Gaussian DOS if charge-carrier diffusion is neglected (but including the carrier density and field dependence of the mobility).

In the absence of diffusion, there is no charge in the device at $V=0$. The current density at finite voltages is then the result of the voltage-dependent transport of a voltagedependent density of injected charges. As a result, the driftonly current density is proportional to $V^{2}$, as given quantitatively by the Mott-Gurney formula [Eq. (1)]. As a result of diffusion, there is already at $V=0$ a space-charge density, $n(x, V=0)$, present in the device. The resulting local resistivity is $\rho(x, V=0)=1 /[e \mu(x, V=0) n(x, V=0)]$ so that in the small-voltage limit the current density varies linearly with $V$ and is (exactly) given by

$$
J_{0}=\frac{1}{\int_{0}^{L} \rho(x, 0) d x} \times V=\frac{1}{\int_{0}^{L} \frac{1}{n(x, 0) \mu(x, 0) e} d x} \times V .
$$

Indeed, all $J(V)$ curves which have been calculated including diffusion show at small voltages this expected Ohmic $J-V$ relationship.

The crossover voltage, $V_{\text {crossover, }}$ between the diffusion and drift-dominated transport regimes may be defined as the voltage at which $J_{\mathrm{MG}}=J_{0}$. For symmetric devices with ideal contacts $\left(n_{1}=n_{2}=\infty\right)$ and a constant mobility and diffusion coefficient, application of Eqs. (9) and (12) leads to ${ }^{27}$

$$
J_{0}=4 \pi^{2} \frac{k_{B} T}{e} \varepsilon \mu_{0} \frac{V}{L^{3}}=2 e n_{\text {center }} \mu_{0} \frac{V}{L} .
$$

The crossover voltage is then given by $V_{\text {crossover }}=32 \pi^{2} / 9$ $\times k_{B} T / e$. At room temperature, $V_{\text {crossover }} \sim 0.9 \mathrm{~V}$, independent of $\varepsilon, \mu$, and $L$. This is consistent with Fig. 6 .

Equation (13) shows that, in the small-voltage limit, the effective conductivity of the device is thus determined by the conductivity in the device center. Strictly speaking, Eq. (13) is only valid for the case of a constant mobility and diffusion coefficient. However, it has already been remarked in Sec. III A that the change in the carrier density distribution across the device upon the introduction of disorder is surprisingly small [see Fig. 4(a)]. In the small-voltage limit, the main effect of the introduction of disorder is therefore expected to be the carrier-density-dependent enhancement of the mobility in the device center. Neglecting the effect of disorder, the carrier concentration in the center of the devices studied in Fig. 4 is $\approx 4 \pi^{2} n_{0} / N_{t} \approx 10^{-5}$. For the case $\sigma /\left(k_{B} T\right)=3$, the carrier density in the center of the device is then deep in the Boltzmann regime [see Fig. 1(a)] so that at small voltages the introduction of disorder only marginally enhances $J_{0}$ above the value obtained when a constant mobility and diffusion coefficient is assumed. In contrast, for the case $\sigma /\left(k_{B} T\right)=6, J_{0}$ is more than 1 order of magnitude larger than the value that is obtained when a constant mobility and dif- 


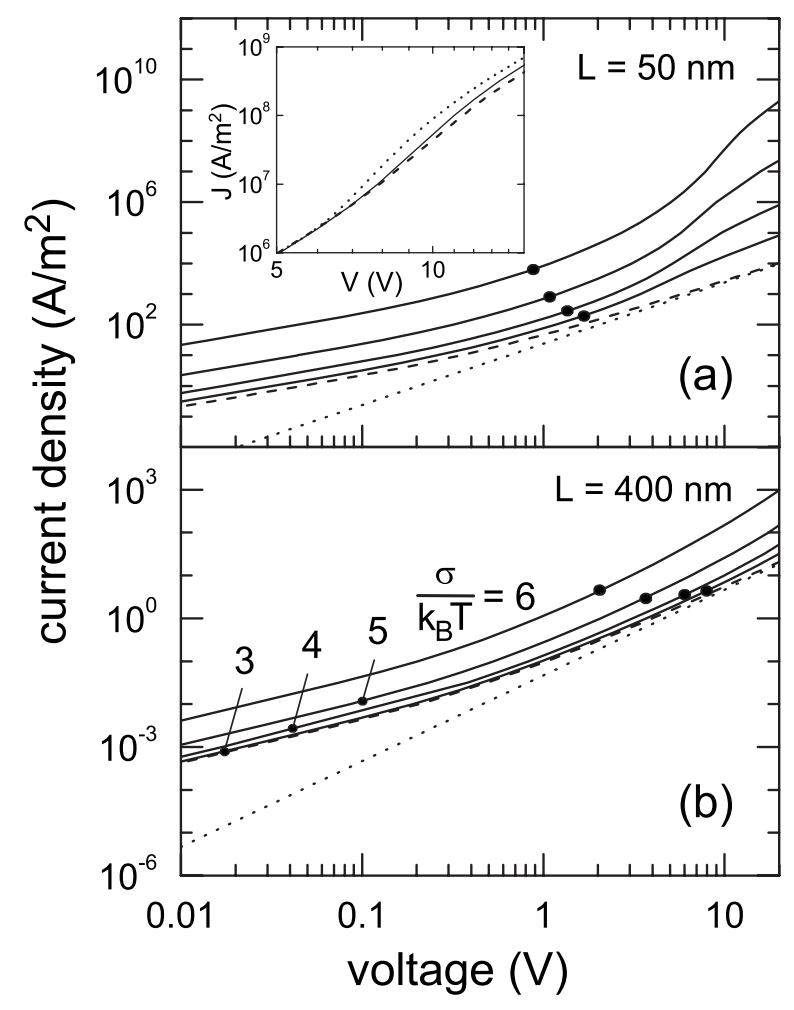

FIG. 7. $J(V)$ curves for transport in a Gaussian DOS in symmetric devices at $T=298 \mathrm{~K}$ with $n_{1}=n_{2}=2.13 \times 10^{26} \mathrm{~m}^{-3}, \mu_{0}=1.0$ $\times 10^{-10} \mathrm{~m}^{2} /(\mathrm{Vs})$, and $\varepsilon_{r}=3$ and with (a) $L=50 \mathrm{~nm}$ and (b) $L$ $=400 \mathrm{~nm}$ devices. The full curves correspond to $\sigma /\left(k_{B} T\right)=6$ (highest $J$ ) to 3 (lowest $J$ ). The filled circles indicate the points (at voltage $V_{2}$ ), at which the slope of the $J(V)$ curves on the doublelogarithmic scale used is equal to 2 . The $J(V)$ curves for the situation without disorder and with and without diffusion are dashed and dotted, respectively. The inset in (a) shows a part of the currentdensity curve for $\sigma /\left(k_{B} T\right)=6$ (full), together with results for lower carrier density and field cutoff values (dashed and dotted curves, respectively; see Appendix A).

fusion coefficient is assumed. The figure shows that only a small part of this current-density enhancement can be understood from the enhancement of the diffusion coefficient in a Gaussian DOS. The largest part of the current-density enhancement can be understood when considering the enhancement of the mobility due to its carrier density dependence. As argued above, we must focus on the mobility in the center of the device, where the carrier concentration is approximately $\sim 10^{-5}$. It can be seen from Fig. 1(a) that this is well outside the Boltzmann regime, leading indeed to an enhancement of the mobility of approximately 1 order of magnitude.

For large voltages, well above $V_{\text {crossover }}$, the carrier density and field dependence of the mobility is seen to significantly enhance the current density above the $J(V)$ curve that is obtained for the case of a constant mobility and diffusion coefficient. For $\sigma /\left(k_{B} T\right)=6$, this enhancement amounts to almost 4 orders of magnitude at $V=20 \mathrm{~V}$. For the devices with $\sigma /\left(k_{B} T\right)=6$ studied in Fig. 6(b), a comparison of the thick full curve with the dashed curve shows that the neglect of diffusion would give rise to errors larger than a factor of two for $V<4 \mathrm{~V}$.

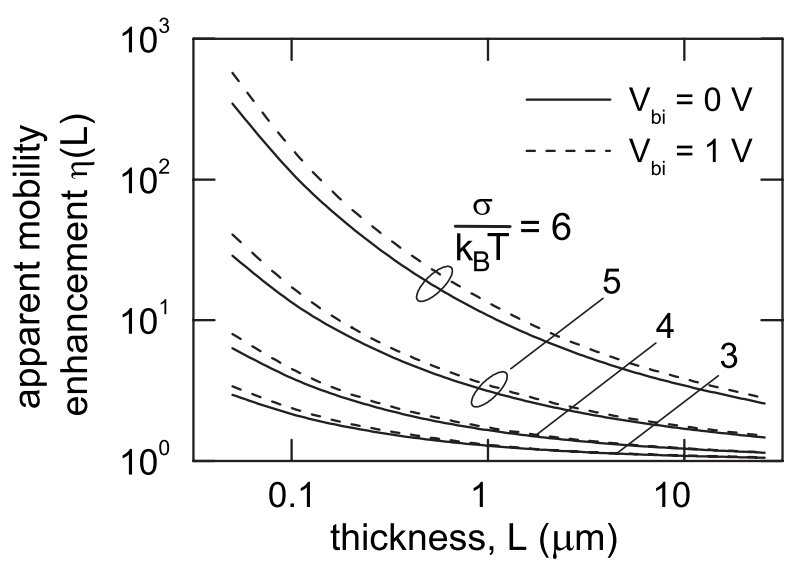

FIG. 8. Apparent mobility enhancement function $\eta(L)$ of the current density as compared to the current density as expected from the Mott-Gurney formula at voltage $V=V_{2}$ at which the slope of the $J(V)$ curves is equal to 2 on a double-logarithmic scale. Results are given for $\sigma /\left(k_{B} T\right)=3$ to 6 for devices with $V_{\mathrm{bi}}=0 \mathrm{~V}$ (full curves) and for devices with $V_{\mathrm{bi}}=1 \mathrm{~V}$ (dashed curves). The device parameters are identical to those given in the captions of Figs. 6 and 9.

It should be noted that all calculations were performed using the same value of $\mu_{0}$, independent of the ratio $\sigma /\left(k_{B} T\right)$. In actual materials, $\mu_{0}$ is expected to decrease with increasing $\sigma$, as described in detail in Refs. 11 and 12. In real devices, the overall effect of enhancing $\sigma$ (all other parameters remaining the same) is therefore a decrease in the current density. As the purpose of showing Figs. 6(a) and 6(b) is to indicate the current-density enhancement as a result of disorder, the actual value of $\mu_{0}$ (to which $J$ is simply proportional) is here of no relevance.

For a series of devices with decreasing layer thickness, for which the carrier densities $n_{1}$ and $n_{2}$ at the electrodes are kept the same, the average carrier density that is present in the device due to diffusion increases. As a result, the effect of the carrier density dependence of the mobility and diffusion coefficient on the current density at small voltages (the linear regime) increases with decreasing layer thickness. This can be seen from Figs. 7(a) and 7(b), which show the current density versus voltage for 50 and $400 \mathrm{~nm}$ devices, respectively, for $\sigma /\left(k_{B} T\right)=3,4,5$, and 6 (full curves). Apart from the layer thickness, all device parameters are equal to those used in Fig. 6. For comparison, $J(V)$ curves for the constant mobility case with (dashed) and without (dotted) diffusion are also given.

Each of the full curves shown in Figs. 7(a) and 7(b) contains a point $\left(V_{2}\right)$ at which the slope (on a double-log scale) is equal to 2 (filled circles, in the range $V \sim 0.8-8 \mathrm{~V}$ ). Conventionally, the voltage range around these points is often viewed as intermediate, situated in between a low-voltage range in which the diffusion contribution to the current density dominates (slope $<2$ ), and a high-voltage range in which the field dependence of the mobility is significant (slope $>2$ ). The current density in this intermediate voltage range is therefore often (incorrectly) assumed to be given by the driftonly Mott-Gurney formula [Eq. (1)], which is then used to obtain the zero-field mobility, $\mu(F=0)$.

Figure 8 gives the ratio $\eta$ of the apparent mobility, $\mu_{\text {app }}$, 
that would follow from the (incorrect) procedure described above and the mobility in the Boltzmann limit, $\mu_{0}$, as a function of the device thickness and for various values of $\sigma /\left(k_{B} T\right)$. This ratio is given by

$$
\eta=\frac{\mu_{\mathrm{app}}}{\mu_{0}}=\frac{J\left(V_{2}\right)}{J_{M G}\left(V_{2}\right)},
$$

where $J\left(V_{2}\right)$ is the actual current density at $V=V_{2}$, and $J_{\mathrm{MG}}\left(V_{2}\right)$ is the current density as given by the Mott-Gurney formula (i.e., neglecting diffusion and with $\mu=\mu_{0}$ ). We emphasize the log scale used in this figure. In the figure, the full lines give the results for the symmetric devices discussed in this section. The figure shows clearly that the application of the conventional approach described above for determining the mobility leads to values of the apparent mobility which are strongly layer thickness dependent. This is a key conclusion of our work.

For very thick devices, diffusion and the carrier density and field enhancement of the mobility may safely be neglected at the voltage $V_{2}$. However, with decreasing thickness, the current density becomes significantly larger than the current density as expected using the Mott-Gurney formula with the mobility as deduced from the current density at large thicknesses. For example, for $100 \mathrm{~nm}$ devices with $\sigma /\left(k_{B} T\right)=6$, which is realistic for PPV-based polymer OLEDs at room temperature, ${ }^{12}$ the enhancement is more than a factor 100. Even for $10 \mu \mathrm{m}$ devices, it is then still approximately a factor of 4 . The large slope of the curves in the thickness range that is most realistic for OLEDs, from 50 to $200 \mathrm{~nm}$, implies that large errors will be made when predicting the thickness dependence of $J(V)$ curves when assuming a constant mobility. Experimentally, this effect was first demonstrated by Blom et al. ${ }^{28}$ for hole transport in PPV-based polymers. We note that in that study the effect of diffusion was neglected, and that the density dependence of the mobility was obtained in a more empirical way, viz., from an analysis of organic field-effect transistor current-voltage curves.

The apparent mobility enhancement, $\eta$, does not only depend on $\hat{\sigma}=\sigma /\left(k_{B} T\right)$ but also on the site density, $N_{t}$. We find that, to a good approximation, this dependence is described by the scaling relation $\eta\left[\hat{\sigma}, N_{t}, L\right] \cong \eta\left(\hat{\sigma}, N_{t} / \beta^{2}, \beta \times L\right)$, where $\beta$ is a dimensionless scaling factor. So the mobility enhancement for a certain device is equal to that for another device which, e.g., is two times thicker and is based on a material with a four times smaller site density. The scaling is exact if the field dependence of the mobility is neglected, as explained in Appendix B. Experimental studies are needed to determine effective values of $N_{t}$ for specific systems. For NRS-PPV and $\mathrm{OC}_{1} \mathrm{C}_{10}-\mathrm{PPV}$, effective values of the intersite distance equal to $a=1.6$ and $1.8 \mathrm{~nm}$ were found in Ref. 12 . That would lead to $N_{t} \approx 0.2 \times 10^{27}$ sites $/ \mathrm{m}^{3}$, approximately a factor of 2 smaller than as assumed in Fig. 8. The scaling relationship given above implies that the enhancement at a thickness $L$ is then equal to the enhancement given in Fig. 8 for the thickness $L / \sqrt{ } 2$. For small-molecule materials, $N_{t}$ might be associated with the number of molecules per $\mathrm{m}^{3}$, which can be around $10^{27} \mathrm{~m}^{-3}$ or even higher.

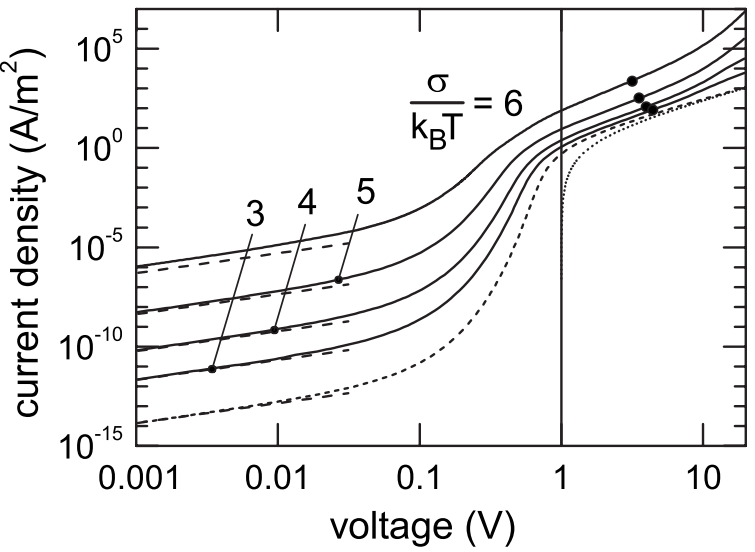

FIG. 9. $J(V)$ curves for transport in a Gaussian DOS in devices at $T=298 \mathrm{~K}$ with $V_{\mathrm{bi}}=1 \mathrm{~V}$ for $\sigma /\left(k_{B} T\right)=3$ to 6 (full curves) and for $L=100 \mathrm{~nm}, \quad N_{t}=4.25 \times 10^{26} \mathrm{~m}^{-3}, \quad n_{1}=0.5 \times N_{t}, \quad \mu_{0}=1.0$ $\times 10^{-10} \mathrm{~m}^{2} /(\mathrm{Vs})$, and $\varepsilon_{r}=3$. The filled circles indicate the points (at voltage $\left.V_{2}\right)$, at which the slope of the $J$ versus $\left(V-V_{\text {bi }}\right)$ curves on a double-logarithmic scale is equal to 2 . The long-dashed lines give the current density in the $V=0$ limit, neglecting the effect of disorder on the mobility and diffusion coefficient [Eq. (15)]. The shortdashed and dotted curves give the current density for devices with a constant mobility and with and without diffusion, respectively.

\section{Current density in asymmetric devices $\left(V_{b i}>0\right)$}

Figure 9 shows how Gaussian disorder affects the $J(V)$ curves for devices with a built-in voltage of $1 \mathrm{~V}$. The Fermi level of the first electrode coincides with the top of the Gaussian DOS so that there is no injection barrier at the first electrode $\left(\varphi_{1}=0 \mathrm{eV}, n_{1} / N_{t}=0.5\right)$ and an injection barrier at the second electrode, $\varphi_{2}$, equal to $1 \mathrm{eV}$. Otherwise, the device parameters are the same as used in Fig. 6. The full curves show the results for devices with Gaussian disorder with $\sigma /\left(k_{B} T\right)=3,4,5$ and 6 . The short-dashed curve shows the result for devices with a constant mobility and diffusion coefficient, and the dotted curve shows the drift-only MottGurney result [Eq. (1)].

In the absence of diffusion, there is no current for $V$ $<V_{\mathrm{bi}}$. As a result of the charge density that is already present in the device due to diffusion, there is actually a finite current density for any finite voltage. For sufficiently small voltages, the $J(V)$ curves are Ohmic with for sufficiently small voltages a current density $J_{0}$ given by Eq. (12). Figure 9 shows that with increasing disorder, $J_{0}$ increases strongly. This may be understood as follows. From Eqs. (11) and (12), it follows that for the special case of a constant mobility and diffusion coefficient, an ideal left contact and a sufficiently large built-in voltage, $J_{0}$ may be expressed as

$$
J_{0}=2 \operatorname{Aen}_{2} \mu_{0} \frac{V}{L},
$$

with $A$ a dimensionless number that depends on $n_{2}$. For the devices studied here, with $V_{\mathrm{bi}}=1 \mathrm{~V}$, this leads to $A=16.8$. Analogous to the case of symmetric devices, studied in 
Sec. III B, this expression for $J_{0}$ is still a very good approximation for systems with a Gaussian DOS, in view of the surprisingly small dependence of the shape of the charge density across the device on the disorder [see Fig. 4(c)]. Equation (15) shows that $J_{0}$ is thus determined by the carrier density at the right contact. This density increases strongly with disorder, e.g., from $n_{2} / N_{t}=1.10 \times 10^{-15}$ for $\sigma /\left(k_{B} T\right)=3$ to $n_{2} / N_{t}=5.47 \times 10^{-10}$ for $\sigma /\left(k_{B} T\right)=6$. In Fig. 9 , the current density that is predicted from Eq. (15) is given by the dashed lines. It may be seen that this almost fully explains the huge increase of $J_{0}$ with increasing $\sigma /\left(k_{B} T\right)$. We note that, in view of the small densities at the exit contact, only for the case $\sigma /\left(k_{B} T\right)=6$ the effect of a small enhancement of the mobility due to its carrier-concentration dependence should be taken into account in order to fully explain $J_{0}$.

The increase in $J_{0}$ with increasing disorder, via the effect of $n_{2}$ on $J_{0}$, should be distinguished from the effect of disorder on the injection limited current density in a device with a large injection barrier at the left contact, first noted by Arkhipov et al. ${ }^{19}$ The authors predicted an enhancement of the injection current due to disorder, resulting from hopping from the Fermi level in the electrodes into the tail of the Gaussian DOS. Whereas the latter effect refers to a hopping process at the electrode interfaces, determined by the injection kinetics, the effect discussed here concerns the influence of the interfacial carrier density on the carrier density throughout the entire device, affecting the bulk transport.

We have investigated to what extent an analysis of the $J(V)$ curves using the Mott-Gurney formula, after the application of a $1 \mathrm{~V}$ built-in voltage correction, would give rise to an apparent mobility enhancement, as found for symmetric devices. At voltages well above $V_{\mathrm{bi}}$, the $J(V)$ curves given in Figs. 6 and 9 coincide after the application of the built-in voltage correction. The agreement becomes worse when $V_{\mathrm{bi}}$ is approached, leading to slightly different values of the voltages, $V_{2}$ (full circles in Fig. 9), at which the slopes of the shifted $J(V)$ curves are equal to two on a double-logarithmic scale. However, the layer thickness and disorder dependent apparent mobility enhancement is very close to that found for symmetric devices, as may be seen from the dashed curves in Fig. 8. For all devices, symmetric and asymmetric, the apparent mobility that would (incorrectly) follow from an analysis of $J(V)$ curves using the Mott-Gurney formula can thus be strongly layer thickness dependent and can vary over many orders of magnitude.

\section{SUMMARY, CONCLUSIONS, AND OUTLOOK}

In this paper, the effect of Gaussian disorder on the voltage dependence of the current density in sandwich-type devices has been studied. The analysis is based on the numerically exact results for the carrier density and field dependence of the mobility given by Pasveer et al. ${ }^{12}$ and it properly includes the carrier density-dependent enhancement of the diffusion coefficient for a Gaussian DOS. ${ }^{6}$ For symmetric and asymmetric devices, we find that conventional analyses of the $J(V)$ curves using the Mott-Gurney relationship [Eq. (1)] can, incorrectly, lead to a very strong layer thickness dependence of the apparent mobility (Fig. 8). Analyses, e.g., of OLED devices, which neglect the effect of disorder on the carrier density and field dependence of the mobility may thus be able to provide a good description of the experimental data for devices with a given layer thickness (as is frequently reported in the literature) but not simultaneously for a range of thicknesses using the same value of $\mu_{0}$. This is a key result of this work.

A detailed analysis has been given of the effect of Gaussian disorder on the carrier density across the device. It has been shown that the effect is surprisingly small, provided that a comparison is made between devices with equal carrier densities at the interfaces (Fig. 4). These results have been used to quantitatively analyze the disorder dependence of the current density at small voltages. The current density is then shown to be proportional to the minimum carrier density in the device. As shown by Fig. 9, the Ohmic current density at small voltages in devices with a large (but fixed) built-in voltage therefore increases significantly with increasing disorder.

A second key result of this work is the development of an efficient drift-diffusion model for charge transport in devices containing disordered organic semiconductors. The model is an extension of an approach developed by Bonham and Jarvis ${ }^{16,17}$ to devices with arbitrary disorder, for which a generalized form of the drift-diffusion equation has been introduced [Eq. (8)]. Although the method has been applied here for the specific case of transport in materials with a Gaussian DOS, it can be applied to any organic semiconductor, provided that the enhancement $(g)$ functions are known. In particular, the method can be extended straightforwardly to systems containing trap states. Such an extension may be envisaged to find practical applications to dye-doped hostguest systems in, e.g., small-molecule OLEDs and to trapcontrolled hole ${ }^{29}$ and electron ${ }^{30-32}$ transport through polymers.

\section{ACKNOWLEDGMENTS}

The authors would like to thank W. F. Pasveer for drawing their attention to Ref. 16 and R. A. J. Janssen for useful discussions. This research was supported by NanoNed, a national nanotechnology program coordinated by the Dutch Ministry of Economic Affairs.

\section{APPENDIX A: MOBILITY AND DIFFUSION COEFFICIENT ENHANCEMENT FUNCTIONS FOR TRANSPORT IN A GAUSSIAN DOS}

The following expressions are used for the mobility and diffusion coefficient enhancement functions: ${ }^{10,11,27}$ 


$$
\begin{gathered}
\left.g_{1}(T, c)=\exp \left[\frac{1}{2}\left(\hat{\sigma}^{2}-\hat{\sigma}\right)(2 c)^{\delta}\right] \quad \text { for } c \leq 0.1, \quad \text { (A }\right) \\
g_{1}(T, c)=g_{1}(T, 0.1) \quad \text { for } \quad c>0.1, \\
g_{2}(T, F)=\exp \left\{0.44\left(\hat{\sigma}^{3 / 2}-2.2\right)\right\}\left[\sqrt{1+0.8\left(\frac{e a F}{\sigma}\right)^{2}}-1\right] \\
\text { for } F \leq 2 \sigma /(e a), \\
\left.g_{2}(T, F)=g_{2}[T, 2 \sigma /(e a)] \text { for } \quad F>2 \sigma /(e a), \quad \text { (A } 4\right)
\end{gathered}
$$

and

$$
\begin{aligned}
g_{3}(T, n) & \equiv \frac{1}{k_{B} T}-\frac{n}{\left.\frac{d n^{\prime}}{d E_{F}}\right|^{n}} \\
& =\frac{n}{\int_{-\infty}^{\infty} N(E) \frac{\exp \left(\frac{E-E_{F}(n, T)}{k_{B} T}\right)}{\left[1+\exp \left(\frac{E-E_{F}(n, T)}{k_{B} T}\right)\right]^{2}} d E .} .
\end{aligned}
$$

In these expressions $c=n / N_{t}$ is the carrier concentration, $a$ $=N_{t}^{-1 / 3}$ is the average intersite distance, $\hat{\sigma}=\sigma /\left(k_{B} T\right)$ is the dimensionless disorder parameter, $\delta$ is given by

$$
\delta=2 \frac{\ln \left(\hat{\sigma}^{2}-\hat{\sigma}\right)-\ln (\ln 4)}{\hat{\sigma}^{2}},
$$

and $E_{F}(c, T)$ is the Fermi energy. In Fig. 1, the dependence of the $g_{1}, g_{2}$, and $g_{3}$ functions on the disorder parameter $\hat{\sigma}$ has been shown.

We have introduced a cutoff carrier concentration, $c_{\text {cutoff }}$ $=0.1$, above which $g_{1}$ is constant, because close to that carrier concentration the compact expression for the mobility enhancement [Eq. (A1)] starts to deviate strongly from the numerically exact master equation result given in Ref. 12 . Instead of a further increase in $g_{1}$ with increasing $c$, as described by Eq. (A1), the mobility increase slows down, or even starts to decrease close to $c=0.1$, depending on the theoretical approach used. ${ }^{11,12,33}$ More theoretical work will be needed to improve the description of the mobility at high carrier densities. In practice, this issue plays only a role for OLEDs with an extremely well injecting electrode, and even in such cases, the thickness of the zone near the electrode in which the carrier concentration is larger than 0.1 is extremely thin (see Fig. 3). Therefore, and due to the high conductivity in this zone, the uncertainty concerning the mobility above $c=0.1$ is expected to be of little influence on the accuracy of calculated $J(V)$ curves of realistic OLEDs. In Sec. III, this is confirmed by making a comparison with the result of a calculation carried out for a much smaller value of the cutoff concentration, $c_{\text {cutoff }}=0.01$ (Fig. 7).

For similar reasons, we have introduced a cutoff field $F_{\text {cutoff }}=2 \sigma /(e a)$ above which $g_{2}$ is constant. Around the cutoff field taken, the enhancement of the mobility with increasing field starts to level off, and at higher fields it even starts to decrease. ${ }^{12}$ This happens when the field is so large that the energies of the down-stream nearest-neighbor sites are displaced by the field over two or three times the width of the Gaussian DOS. In such a case, the mobility is not anymore thermally activated and a further increase in the field does not lead to an increase of the current density. The mobility then shows a weak (linear) decrease with increasing field. In principle, it would be possible to adapt the function $g_{2}$ by taking the more complex field dependence of the mobility, obtained in Ref. 12, into account. However, in practice, the maximum voltages typically applied across OLEDs usually do not give rise to fields close to the cutoff field, as shown in more detail in Sec. III. Only in the case of well injecting electrodes, high fields can occur in very thin interface regions as a result of the interaction between the space charge in the device and its image charge in the electrode. It is presently not yet clear whether the continuum approach that is followed in this paper provides a fully adequate treatment of transport through such space-charge layers, in which there is a very strong field and carrier density gradient. Therefore, the use of a more complex form of $g_{2}$ is presently not justified.

The sensitivity to the cutoff in the parametrization used for the enhancement functions $g_{1}$ and $g_{2}$ has been investigated for the symmetric devices studied in Sec. III B for which the effect on the $J(V)$ curves is expected to be largest, viz., those with $L=50 \mathrm{~nm}$ and $\sigma /\left(k_{B} T\right)=6$. The inset in Fig. 7 (a) shows that the effect of taking $c_{\text {cutoff }}=0.01$, instead of 0.1 (dashed curve), or of taking $F_{\text {cutoff }}=1.5 \times \sigma /(e a)$, instead of $2 \sigma /(e a)$ (dotted curve), leads only for $V>7 \mathrm{~V}$ to a very small although noticeable deviation. This confirms the appropriateness of the parametrization scheme used.

The enhancement $g_{1}$ of the mobility sets in at a carrier concentration that decreases with increasing disorder parameter $\sigma /\left(k_{B} T\right)$. The boundary concentration, $c^{*}$, between the low-concentration Boltzmann regime, in which the mobility is constant, and the high-concentration regime in which it is enhanced may be defined as the concentration at which $g_{1}$ is equal to 2. In Appendix A of Ref. 11 it was proven that $c^{*}$ is equal to the carrier concentration for which the Fermi energy is equal to the thermal equilibrium energy $E_{0}=-\sigma^{2} /\left(k_{B} T\right)$, leading to

$$
c^{*}=c\left(E_{F}=E_{0}\right)=\frac{1}{2} \exp \left(-\frac{1}{2} \hat{\sigma}^{2}\right) .
$$

Analogously, one may define another characteristic carrier concentration, $c^{* *}$, as the concentration at which the enhancement $g_{3}$ of the diffusion coefficient is equal to 2 . We have found that $c^{* *}$ is equal to the carrier concentration for which the Fermi energy is equal to half the thermal equilibrium energy, i.e., $c^{* *}=c\left(E_{F}=E_{0} / 2\right)$. We are not aware of a published proof of this result. We use Eq. (A5) and apply the transformation $U=E / \sigma+\hat{\sigma} / 2$, 


$$
\begin{aligned}
g_{3}\left(E_{F}=-E_{0} / 2\right)= & \frac{\int_{-\infty}^{\infty} \frac{\exp \left(-\frac{E^{2}}{2 \sigma^{2}}\right)}{1+\exp \left(\frac{E}{k_{B} T}+\frac{\sigma^{2}}{2\left(k_{B} T\right)^{2}}\right)} d E}{\int_{-\infty}^{\infty} \exp \left(-\frac{E^{2}}{2 \sigma^{2}}\right) \frac{\exp \left(\frac{E}{k_{B} T}+\frac{\sigma^{2}}{2\left(k_{B} T\right)^{2}}\right)}{\left[1+\exp \left(\frac{E}{k_{B} T}+\frac{\sigma^{2}}{2\left(k_{B} T\right)^{2}}\right)\right]^{2}} d E}=\frac{\int_{-\infty}^{\infty} \exp \left(-\frac{U^{2}}{2}\right) \exp \frac{(U \hat{\sigma} / 2)}{(1+\exp (U \hat{\sigma}))} d U}{\int_{-\infty}^{\infty}\left(-\frac{U^{2}}{2}+\frac{U \hat{\sigma}}{2}\right) \exp (U \hat{\sigma}) /[1+\exp (U \hat{\sigma})]^{2} d U} \\
= & \frac{\int_{-\infty}^{\infty} \exp \left(-\frac{U^{2}}{2}\right)\left[\exp \left(\frac{U \hat{\sigma}}{2}\right)+\exp \left(-\frac{U \hat{\sigma}}{2}\right)\right] / \cosh ^{2}\left(\frac{U \hat{\sigma}}{2}\right) d U}{\int_{-\infty}^{\infty} \exp \left(-\frac{U^{2}}{2}+\frac{U \hat{\sigma}}{2}\right) / \cosh ^{2}\left(\frac{U \hat{\sigma}}{2}\right) d U} .
\end{aligned}
$$

The enumerator of the last expression may be written as a sum of two equal contributions that result from the two terms in between the square brackets, and each of these contributions is equal to the denominator. That proofs that $g_{3}\left(E_{F}=E_{0} / 2\right)=2$. As a result,

$$
c^{* *}=c\left(E_{F}=E_{0} / 2\right)=\frac{1}{\sqrt{8 \pi}} \exp \left(-\frac{1}{8} \hat{\sigma}^{2}\right) \int_{-\infty}^{\infty} \frac{\exp \left(-\frac{E^{2}}{2}\right)}{\cosh \left(\frac{\hat{\sigma}}{2} E\right)} d E .
$$

It follows that $c^{* *}$ is much larger than $c^{*}$, as can also be seen from Figs. 1(a) and 1(c). From the approximate dependence of $g_{1}$ on the Fermi energy, given by Eq. (28) in Ref. 11, it follows that $g_{1}\left(c^{* *}\right) \approx 1 / c^{* *}$. The degree to which this interesting relationship is valid is apparent from the position of the open circles in Fig. 1(a) with respect to the full curves.

\section{APPENDIX B: DERIVATION OF THE DIMENSIONLESS \\ TRANSPORT EQUATION AND OF THE EXPRESSIONS FOR THE DIMENSIONLESS CURRENT DENSITY, VOLTAGE, AND POSITION}

Following Bonham and Jarvis, ${ }^{16}$ the drift-diffusion equation [Eq. (6)] may be written in dimensionless form [Eq. (8)] by first defining a dimensionless carrier density, field, applied voltage, position, and current density,

$$
\begin{gathered}
\gamma \equiv \frac{e^{2} L^{2}}{\varepsilon k_{B} T} n, \\
E \equiv \frac{e L}{k_{B} T} F, \\
u \equiv \frac{e}{k_{B} T} V, \\
s \equiv \frac{x}{L}, \\
i \equiv\left(\frac{e}{k_{B} T}\right)^{2} \frac{L^{3}}{\varepsilon \mu_{0}} J .
\end{gathered}
$$

Equation (6) can then be rewritten in a compact form as

$$
i=g_{1} g_{2} \gamma E-g_{1} g_{2} g_{3} \frac{d \gamma}{d s},
$$

and the Poisson equation [Eq. (7)] is then given by $d E / d s$ $=\gamma$. Equation $(\mathrm{B} 6)$ can then be transformed to

$$
i=g_{1} g_{2} \gamma E-g_{1} g_{2} g_{3} \gamma \frac{d \gamma}{d E} .
$$

Bonham and Jarvis ${ }^{16}$ showed that it is useful to make use of a second transformation to scaled dimensionless field and density parameters $f$ and $y$, defined as

$$
f \equiv \frac{E}{i^{1 / 3}}
$$

and

$$
y \equiv \frac{\gamma}{i^{2 / 3}} .
$$

Substitution of these expressions in Eq. (B7) leads then to the dimensionless transport equation [Eq. (8)].

The current density, voltage, and carrier density across the device can in the following way be obtained from the solutions $y(f)$ of Eq. (8). From Eqs. (B8) and (B9) and the dimensionless Poisson equation $d E / d s=\gamma$, it follows that $i^{2 / 3}$ $=[1 / y(f)] d E / d s=[1 / y(f)] i^{1 / 3} d f / d s$ so that $i^{1 / 3}$ $=[1 / y(f)] d f / d s$. Integration over $s$ then yields 


$$
i=\left(\int_{f_{1}}^{f_{2}} \frac{1}{y(f)} d f\right)^{3}
$$

as given already in Ref. 16. The dimensionless electrochemical potential difference (voltage, $u$ ) may be written as a sum of the dimensionless built-in voltage, $v_{\mathrm{bi}}$, plus the electrostatic potential difference, given by $\int E \cdot d s$. Using Eqs. (B8) and (B9) and the Poisson equation, it follows then straightforwardly that $u$ is given by

$$
u=v_{\mathrm{bi}}+\int_{f_{1}}^{f_{2}} \frac{f}{y(f)} d f
$$

where the dimensionless built-in voltage is by definition equal to

$$
v_{\mathrm{bi}} \equiv \frac{\varphi_{2}-\varphi_{1}}{k_{B} T}
$$

We note that Eq. (B11) is equivalent to Eq. (17) in Ref. 16 for the special case of a constant mobility, but that Eq. (B11) is more general. Using the Poisson equation and Eqs. (B8) and (B9) to write $d s=d E / \gamma=i^{1 / 3} d f /\left(i^{2 / 3} y\right)$, integration yields the position $s$ at field $f(s)$,

$$
s(f)=\int_{f_{1}}^{f} \frac{1}{i^{1 / 3} y\left(f^{\prime}\right)} d f^{\prime} .
$$

Equations (B10), (B11), and (B13) are valid for any system, including systems with a carrier density and field-dependent mobility and diffusion coefficient, as their derivation does not involve the drift-diffusion equation.

As explained in Sec. II B, for obtaining type-II solutions it is often convenient to use the inflection point in the $y(f)$ curve as a starting point for the determination of the full $y(f)$ function. From Eq. (8), it follows that for a given chosen value of $f_{i}$, the value of $y_{i}$ can be obtained from the equation

$$
\begin{aligned}
& g_{1}\left(y_{i}\right) g_{2}\left(f_{i}\right) y_{i}^{3}+\left.\frac{d \ln \left[g_{2}(F)\right]}{d F}\right|_{f_{i}} \frac{d F}{d f} y_{i}^{2} \\
& +\left(1+\left.\frac{d \ln \left[g_{1}(y) g_{3}(y)\right]}{d y}\right|_{y_{i}} y_{i}-g_{1}\left(y_{i}\right) g_{2}\left(F_{i}\right)\right. \\
& \left.\quad \times\left|\frac{d \ln \left[g_{3}(y)\right]}{d y}\right|_{y_{i}} f_{i} y_{i}^{2}\right) \times\left(\frac{f_{i} y_{i}}{g_{3}\left(y_{i}\right)}-\frac{1}{g_{1}\left(y_{i}\right) g_{2}\left(f_{i}\right) g_{3}\left(y_{i}\right)}\right) \\
& =0
\end{aligned}
$$

where [from Eqs. (B2), (B5), and (B8)] $d F / d f$ $=\left[(e J) /\left(k_{B} T \varepsilon \mu_{0}\right)\right]^{1 / 3}$. Furthermore, it has been argued in Sec. II B that type-II solutions are more conveniently obtained from functions $f(y)$ than from functions $y(f)$. The current density can then be obtained by rewriting Eq. (B10) using Eq. (8),

$$
\begin{aligned}
i & =\left(\int_{f_{1}}^{f_{2}} \frac{1}{y(f)} d f\right)^{3}=\left(\int_{f_{1}}^{f_{2}} \frac{1}{y(f)} \frac{d f}{d y} d y\right)^{3} \\
& =\left(\int_{y_{1}}^{y_{2}} \frac{g_{1} g_{2} g_{3}}{g_{1} g_{2} y f(y)-1} d y\right)^{3} .
\end{aligned}
$$

Expressions for the voltage and position, obtained in a fully analogous way from Eqs. (B11) and (B13), are

$$
u=v_{\mathrm{bi}}+\int_{y_{2}}^{y_{1}} \frac{g_{1}(y) g_{2}[f(y)] g_{3}(y) f(y)}{1-g_{1}(y) g_{2}[f(y)] y f(y)} d y,
$$

and

$$
s(y)=\int_{y}^{y_{1}} \frac{g_{1}\left(y^{\prime}\right) g_{2}\left[f\left(y^{\prime}\right)\right] g_{3}\left(y^{\prime}\right)}{i^{1 / 3}\left[1-g_{1}\left(y^{\prime}\right) g_{2}\left[f\left(y^{\prime}\right)\right] y^{\prime} f\left(y^{\prime}\right)\right]} d y^{\prime} .
$$

In Sec. III B, a scaling relationship has been given for the apparent mobility enhancement function, $\eta$, in a device with a Gaussian DOS of the form $\eta\left[\hat{\sigma}, N_{t}, L\right] \cong \eta\left(\hat{\sigma}, N_{t} / \beta^{2}, \beta\right.$ $\times L$ ). Here, $\beta$ is a dimensionless scaling factor. This scaling can be understood as follows. The mobility and diffusion coefficient enhancement functions $g_{1}$ and $g_{3}$ are a function of the local carrier concentration, $c$. From Eqs. (B1) and (B9), $c \propto y /\left(N_{t} L^{2}\right)$. Therefore, a change in the system under which the product $N_{t} L^{2}$ remains constant does not change the solution of the dimensionless transport equation, Eq. (8). It can be seen from Eq. (B1) that also the boundary conditions if expressed in terms of $n_{1} / n_{0}$ and $n_{2} / n_{0}$ will then not change. The two situations are therefore fully equivalent with the same dimensionless currents $i$, and [using Eq. (B5)] with real current densities $J$ that differ at any voltage precisely by a factor $L^{3}$.

\section{APPENDIX C: ITERATIVE METHODS FOR SOLVING THE DRIFT-DIFFUSION EQUATION}

In this Appendix, two alternative iterative methods are described for obtaining solutions of the drift-diffusion equation: (i) method $\mathrm{A}$, for fixed values of $f_{\min }$ or $f_{i}$, and (ii) method B, for fixed values of $J$.

Method A: This method involves the following steps:

Step 1. Choose for a given value of $f_{\min }$ or $f_{i}$ a trial current density, $J_{1}$.

Step 2. Calculate a new current density, $J_{2}$, using the method outlined in Sec. II.

Step 3. Use this new current density as a second trial current density.

Step 4. Repeat this until convergence has been reached. The approach makes use of the fact that for a fixed $f_{\min }$ or $f_{i}$ a large positive (negative) deviation of the "trial" value of the current density, $J_{1}$, from the actual solution leads to a smaller positive (negative) deviation of the resulting calculated current density, $J_{2}$. This is analyzed below in more detail.

For the case of a constant mobility, method A leads to fast convergence when the starting value of $J_{1}$ is sufficiently close to the solution $J$. However, that is not always true for the case of a Gaussian DOS. In such a case, method B, 


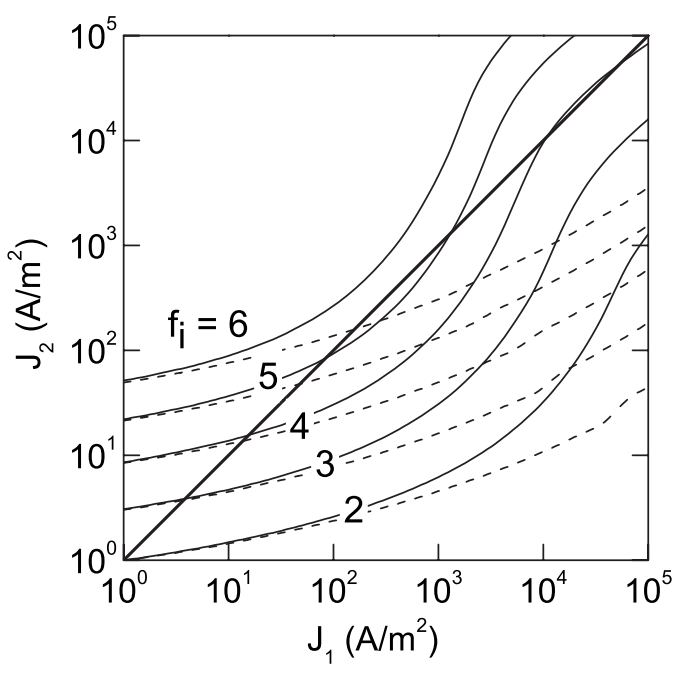

FIG. 10. Calculated current density $J_{2}$ as a function of the assumed current density $J_{1}$ for various values of the dimensionless field at the inflection point, $f_{i}$, for a device with $\sigma /\left(k_{B} T\right)=4$ and $V_{\mathrm{bi}}=1$, and further device parameters as given in the text. The full and dashed curves follow from calculations with and without the field enhancement of the mobility, respectively. For any chosen value of $J_{1}$, Eq. (8) is solved by varying $f_{i}$ until $J_{2}=J_{1}$.

described below, must be used. As an example, the situation for a device at $T=298 \mathrm{~K}$, with $L=100 \mathrm{~nm}, \mu_{0}=1.0$ $\times 10^{-10} \mathrm{~m}^{2} /(\mathrm{Vs}), \quad \varepsilon_{r}=3, \quad N_{t}=4.25 \times 10^{26} \mathrm{~m}^{-3}, \quad \varphi_{1}=0 \mathrm{eV}$, $\varphi_{2}=1 \mathrm{eV}$, and $\sigma /\left(k_{B} T\right)=4$, is analyzed. For all voltages, the solutions are of type II. The thin full curves in Fig. 10 show how for various values of the field $f_{i}$ at the inflection point the calculated current density, $J_{2}$, depends on the value of a chosen trial value of the current density, $J_{1}$. It follows from the figure that for small values of $f_{i}$, e.g., $f_{i}=3$, method $\mathrm{A}$ will provide fast convergence, as the slope of the thin $f_{i}=3$ line is smaller than 1 near the point where $J_{1}=J_{2}$ (thick line). In contrast, for larger values of $f_{i}$, convergence is not always reached. For $f_{i}=5$, e.g., there are two solutions in the frame of the figure, of which the solution at the highest current density $\left(\sim 10^{3} \mathrm{~A} \mathrm{~m}^{-2}\right)$ cannot be found using method $\mathrm{A}$. This is due to the fact that the slope of the thin $f_{i}=5$ line is larger than 1 near the point where $J_{1}=J_{2}$.

Method B: This method involves the following steps:

Step 1 . Select the value of the current density $J$ for which a solution is to be found.

Step 2. Calculate the boundary conditions $y_{1}$ and $y_{2}$ using Eqs. (5), (B1), (B5), and (B9).

Step 3. Calculate the current density $J^{\prime}$ at two trial values for $f$ at the minimum or inflection point, $f_{\text {start }}^{\prime}$. First, the corresponding value of $y_{\text {start }}^{\prime}$ is calculated by numerically solving Eq. (8), with $d f / d y=0$ in the case of a type-I solution, or Eq. (B14) in the case of a type-II solution. Then, the functions $f_{+}(y)$ and $f_{-}(y)$ are calculated in the intervals $\left[y_{2}, y_{\text {start }}\right]$ and $\left[y_{\text {start }}, y_{1}\right]$, respectively, by numerical integration using Eq. (8). Finally, these $f$ functions are used to calculate $J^{\prime}$ from Eqs. (B5) and (B15).

Step 4. Determine from the resulting two current densities $J^{\prime}$ a first prediction of $f_{\text {start }}$ by interpolation or extrapolation. Use is made of the fact that, for a given value of $J, J^{\prime}$ is a monotonic and increasing function of $f_{\text {start }}$.
Step 5. Calculate the current density for this predicted value of $f_{\text {start }}$ and use that (and all or part of the previously calculated current densities) to obtain a next prediction.

Step 6. Repeat step 5 until a sufficiently accurate result is obtained.

The resulting self-consistent $f(y)$ function can be used to calculate from Eqs. (B3), (B12), and (B16) the voltage $V$ and (if of interest) to calculate using Eqs. (B1), (B9), and (B17) the carrier density across the device. As emphasized in Appendix D, special care must be taken when $V$ is very close to the transition voltage $V^{*}$, as the minimum or inflection point reside then outside the device.

We have used method B for all our calculations. The failure (under some conditions) of the slightly more simple method A is due to the field dependence of the mobility in a Gaussian DOS. That may be seen from the fact that the full curves in Fig. 10 have for certain current density ranges a slope larger than 1, whereas the dashed curves (which are obtained when setting $g_{2}$ equal to 1) all have a slope smaller than 1 at the point of crossing the thick diagonal line.

\section{APPENDIX D: THE TRANSITION FROM TYPE-I TO TYPE-II SOLUTIONS}

In systems with a finite value of the built-in voltage, a transition from a type-I to a type-II solution occurs when the voltage exceeds a certain value. The following method, illustrated here for the case of a constant mobility, can be used to determine the transition voltage, $V^{*}$. First, the curve $Y(f)$ that separates the two parts of the diagram shown in Fig. 2 is calculated. $Y(f)$ can be obtained numerically by integration using Eq. (8) (with all $g$ functions equal to 1), starting at a point $f_{\text {start }} \gg 1$, and using that then $Y\left(f_{\text {start }}\right) \approx 1 / f_{\text {start. As } Y}$ varies monotonically with $f$, it is also possible to define the inverse function, $F(y)$. The transition current density, $i^{*}$, is the solution of the integral equation

$$
i^{*}=\left(\int_{f_{1}\left(i^{*}\right)}^{f_{2}\left(i^{*}\right)} \frac{1}{Y(f)} d f\right)^{3},
$$

with

$$
f_{1}\left(i^{*}\right)=F\left(\frac{\gamma_{1}}{\left(i^{*}\right)^{2 / 3}}\right) \text { and } f_{2}\left(i^{*}\right)=F\left(\frac{\gamma_{2}}{\left(i^{*}\right)^{2 / 3}}\right) .
$$

Here, $V^{*}$ can then be calculated using Eqs. (B3) and (B11). Figure 11 shows the dependence of $V^{*}$ on the dimensionless carrier densities at the interfaces, $\gamma_{1}$ and $\gamma_{2}$, for a device with the same thickness $(L=100 \mathrm{~nm})$ and relative permittivity $\left(\varepsilon_{r}=3\right)$ as taken in Fig. 3, also at $T=298 \mathrm{~K}$. The two filled circles correspond to the boundary conditions for which $n(x)$ curves are given in Figs. 3(a) and 3(b). For symmetric devices $\left(\gamma_{1}=\gamma_{2}\right)$, there is at any voltage a minimum in the carrier density so that $V^{*}$ is infinite. When $\gamma_{2}$ is smaller than $\gamma_{1}$, but still sufficiently large, $V^{*}$ is finite (upper part of the triangle). When $\gamma_{2}$ is sufficiently small and not too close to $\gamma_{1}, V^{*}=0$ (part of the triangle at and below the $V^{*}=0$ contour).

No distinct physical effect is expected at the type-I to type-II transition. This may, e.g., be seen from Fig. 3. In that 


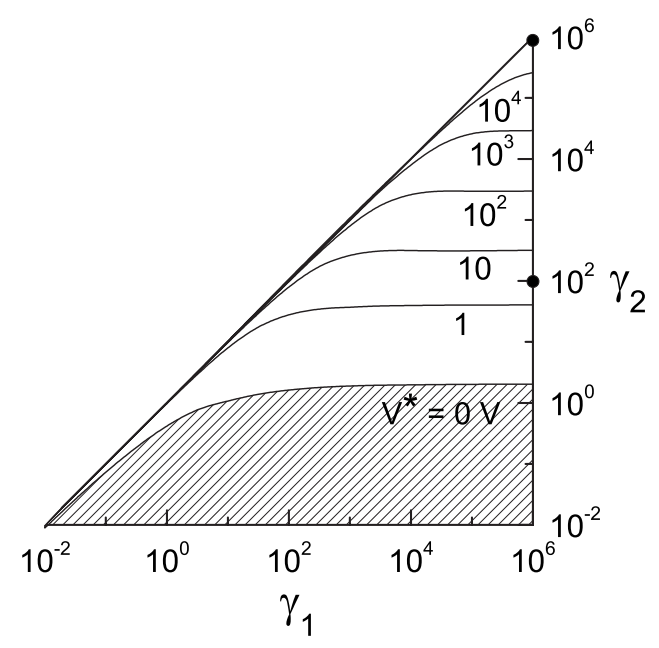

FIG. 11. The transition voltage $V^{*}$ between solution types I and II as a function of the dimensionless carrier density at the two electrodes, $\gamma_{1}$ and $\gamma_{2}$, for the devices with $L=100 \mathrm{~nm}$ and $\varepsilon_{r}=3$ at $T=298 \mathrm{~K}$. The two filled circles indicate the boundary conditions used in Figs. 4(a) and 4(b).

figure, the thick dashed curve connects the end points $\left(y_{2}, f_{2}\right)$ of the density versus field segments which are a solution of Eq. (8) for the system discussed in Fig. 4(b). The curve shows no discontinuity at the boundary between the type-I and type-II regions. However, close inspection of Fig. 3 shows that the transition is mathematically slightly more complicated than one might have anticipated. The figure shows that at low voltages, the $f_{\min }$ value of the solutions $y(f)$ is smaller than $f_{2}$ so that the carrier density minimum falls inside the device. In contrast, very close to the transition voltage, $V^{*}$, at which the $y(f)$ solution switches from the upper to the lower part of the diagram, the $f_{\min }$ value of the solutions $y(f)$ is larger than $f_{2}$ so that the minimum of the carrier density falls outside the device. This follows from the fact that $f_{2}$ has a finite value at the transition $\left(f_{2}=6.85\right.$ for the example shown), whereas $f_{\min }$ approaches infinity when $V$ approaches $V^{*}$. At $V^{*}$, the solution $y(f)$ coincides (in the interval $\left[f_{1}, f_{2}\right]$ for which it is defined) with the curve that separates the two parts of the diagram shown in Fig. 3. In a certain voltage interval just below $V^{*}$, the proper solution thus does not show a carrier density minimum within the device, but outside the device. Similarly, in a certain voltage interval just above $V^{*}$, the proper solution does not show an inflection point within the device, but outside the device. As a result of the strong change of the slope of the $y(f)$ curves close to the second electrode, the widths of these voltage intervals at either side of $V^{*}$ is in many cases extremely small and of little practical importance. For the example discussed above, their widths are less than $0.1 \mathrm{~V}$.
${ }^{1}$ Organic Light-Emitting Devices, edited by K. Müllen and U. Scherf (Wiley, Weinheim, 2006).

${ }^{2}$ P. M. Borsenberger and D. S. Weiss, Organic Photoreceptors for Xerography (Dekker, New York, 1998).

${ }^{3}$ R. A. J. Janssen, J. C. Hummelen, and N. S. Sariciftci, MRS Bull. 30, 30 (2005).

${ }^{4}$ P. W. M. Blom and M. C. J. M. Vissenberg, Mater. Sci. Eng., R. 27, 3 (2000); A. B. Walker, A Kambili, and S. J. Martin, J. Phys.: Condens. Matter 14, 9825 (2002).

${ }^{5}$ M. A. Lampert and P. Mark, Current Injection in Solids (Academic, New York, 1970).

${ }^{6}$ Y. Roichman and N. Tessler, Appl. Phys. Lett. 80, 1948 (2002); Y. Preezant and N. Tessler, J. Appl. Phys. 93, 2059 (2003).

${ }^{7}$ C. Tanase, E. J. Meijer, P. W. M. Blom, and D. M. de Leeuw, Phys. Rev. Lett. 91, 216601 (2003).

${ }^{8}$ C. Tanase, P. W. M. Blom, and D. M. de Leeuw, Phys. Rev. B 70, 193202 (2004).

${ }^{9}$ M. C. J. M. Vissenberg and M. Matters, Phys. Rev. B 57, 12964 (1998).

${ }^{10}$ Y. Roichman, Y. Preezant, and N. Tessler, Phys. Status Solidi A 201, 1246 (2004).

${ }^{11}$ R. Coehoorn, W. F. Pasveer, P. A. Bobbert, and M. A. J. Michels, Phys. Rev. B 72, 155206 (2005).

${ }^{12}$ W. F. Pasveer, J. Cottaar, C. Tanase, R. Coehoorn, P. A. Bobbert, P. W. M. Blom, D. M. de Leeuw, and M. A. J. Michels, Phys. Rev. Lett. 94, 206601 (2005).

${ }^{13} \mathrm{~S}$. Selberherr, Analysis and Simulation of Semiconductor Devices (Springer, Vienna, 1984).

${ }^{14}$ P. S. Davids, I. H. Campbell, and D. L. Smith, J. Appl. Phys. 82,
6319 (1997); J. C. Scott, P. J. Brock, J. R. Salem, S. Ramos, G. G. Malliaras, S. A. Carter, and L. Bozano, Synth. Met. 111-112, 289 (2000); J. Staudigel, M. Stößel, F. Steuber, and J. Simmerer, J. Appl. Phys. 86, 3895 (1999); D. Berner, H. Houili, W. Leo, and L. Zuppiroli, Phys. Status Solidi A 202, 9 (2005).

${ }^{15}$ L. J. A. Koster, E. C. P. Smits, V. D. Mihailetchi, and P. W. M. Blom, Phys. Rev. B 72, 085205 (2005); S. Scheinert and G. Paasch, Phys. Status Solidi A 201, 1263 (2004), and references therein.

${ }^{16}$ J. S. Bonham and D. H. Jarvis, Aust. J. Chem. 30, 705 (1977).

${ }^{17}$ J. S. Bonham and D. H. Jarvis, Aust. J. Chem. 31, 2103 (1978).

${ }^{18}$ J. Campbell Scott and G. G. Malliaras, Chem. Phys. Lett. 299, 115 (1999).

${ }^{19}$ V. I. Arkhipov, E. V. Emelianova, Y. H. Tak, and H. Bässler, J. Appl. Phys. 84, 4438 (1998).

${ }^{20}$ I. I. Fishchuk, V. I. Arkhipov, A. Kadashchuk, P. Heremans, and H. Bässler, Phys. Rev. B 76, 045210 (2007).

${ }^{21}$ L. B. Schein, D. Glatz, and J. C. Scott, Phys. Rev. Lett. 65, 472 (1990); L. Th. Pautmeier, J. C. Scott, and L. B. Schein, Chem. Phys. Lett. 197, 568 (1992).

${ }^{22}$ K. D. Meisel, H. Vocks, and P. A. Bobbert, Phys. Rev. B 71, 205206 (2005).

${ }^{23}$ S. L. M. van Mensfoort, S. I. E. Vulto, R. A. J. Janssen, and R. Coehoorn, following paper, Phys. Rev. B 78, 085208 (2008).

${ }^{24}$ H. Bässler, Phys. Status Solidi B 175, 15 (1993).

${ }^{25}$ D. M. Pai, J. Chem. Phys. 52, 2285 (1970); D. M. Pai, J. F. Janus, and M. Stolka, J. Phys. Chem. 88, 4717 (1984).

${ }^{26}$ O. Rubel, S. D. Baranovskii, P. Thomas, and S. Yamasaki, Phys. Rev. B 69, 014206 (2004). 
${ }^{27}$ R. de Levie and H. Moreira, J. Membr. Biol. 9, 241 (1972); R. de Levie, N. G. Seidah, and H. Moreira, ibid. 10, 171 (1972).

${ }^{28}$ P. W. M. Blom, C. Tanase, D. M. de Leeuw, and R. Coehoorn, Appl. Phys. Lett. 86, 092105 (2005).

${ }^{29}$ A. J. Campbell, D. D. C. Bradley, and D. G. Lidzey, J. Appl. Phys. 82, 6326 (1997).

${ }^{30}$ P. E. Burrows and S. R. Forrest, Appl. Phys. Lett. 64, 2285 (1994).
${ }^{31}$ Z. Shen, P. E. Burrows, V. Bulovic, D. M. McCarty, M. E. Thompson, and S. R. Forrest, Jpn. J. Appl. Phys., Part 2 35, L401 (1996).

${ }^{32}$ M. M. Mandoc, B. de Boer, G. Paasch, and P. W. M. Blom, Phys. Rev. B 75, 193202 (2007).

${ }^{33}$ J. Zhou, Y. C. Zhou, J. M. Zhao, C. Q. Wu, X. M. Ding, and X. Y. Hou, Phys. Rev. B 75, 153201 (2007). 